\title{
A Polynomial Kernel for Trivially Perfect Editing*
}

\author{
Pål Grønås Drange Michał Pilipczuk $^{\ddagger}$
}

October 9, 2018

\begin{abstract}
We give a kernel with $O\left(k^{7}\right)$ vertices for Trivially PERfECT EDITING, the problem of adding or removing at most $k$ edges in order to make a given graph trivially perfect. This answers in affirmative an open question posed by Nastos and Gao [27], and by Liu et al. [24]. Our general technique implies also the existence of kernels of the same size for related Trivially PERfECT Completion and Trivially Perfect Deletion problems. Whereas for the former an $O\left(k^{3}\right)$ kernel was given by Guo [19], for the latter no polynomial kernel was known.

We complement our study of Trivially PERFECT EDITINg by proving that, contrary to Trivially Perfect Completion, it cannot be solved in time $2^{o(k)} \cdot n^{O(1)}$ unless the Exponential Time Hypothesis fails. In this manner we complete the picture of the parameterized and kernelization complexity of the classic edge modification problems for the class of trivially perfect graphs.
\end{abstract}

\section{Introduction}

Graph modification problems form an important subclass of discrete computational problems, where the task is to modify a given graph using a constrained number of modifications in order to make it satisfy some property $\Pi$, or equivalently belong to the class $\mathcal{G}$ of graphs satisfying $\Pi$. Well-known examples of graph modification problems include Vertex Cover, Cluster Editing, Feedback Vertex Set, Odd Cycle Transversal, and Minimum Fill-In. The systematic study of graph modification problems dates back to early 80s and the work of Yannakakis [28], who showed that there is a dichotomy for the vertex deletion problems: unless a graph class $\mathcal{G}$ is trivial (finite or co-finite), the problem of deleting the least number of vertices to obtain a graph from $\mathcal{G}$ is NP-hard. However, when, in order to obtain a graph from $\mathcal{G}$, we are to modify the edge set of the graph instead of the vertex set, there are three natural classes of problems: deletion problems (deleting the least number of edges), completion problems (adding the least number of edges) and editing problems (performing the least number of edge additions or deletions). For neither of these, any complexity dichotomy in the spirit of Yannakakis' result is known. Indeed, in [28] Yannakakis states

It [...] would be nice if the same kind of techniques could be applied to the edge-deletion problems. Unfortunately we suspect that this is not the case - the reductions we found for the properties considered [...] do not seem to fall into a pattern.

- Mihalis Yannakakis

Even though for edge modification problems there is no general P vs. NP classification known, much can be said about their parameterized complexity. Recall that a parameterized problem is called fixedparameter tractable if it can be solved in time $f(k) \cdot n^{O(1)}$ for some computable function $f$, where $n$ is the size of the input and $k$ is its parameter. In our case, the natural parameter $k$ is the allowed number of modifications. Cai [5] made a simple observation that for all the aforementioned graph modification problems there is a simple branching algorithm running in time $c^{k} n^{O(1)}$ for some constant $c$, as long as $\mathcal{G}$ is

*The research leading to these results has received funding from the European Research Council under the European Union's Seventh Framework Programme (FP/2007-2013) / ERC Grant Agreement n. 267959. M. Pilipczuk is currently holding a post-doc position at Warsaw Center of Mathematics and Computer Science, and his research is supported by Polish National Science Centre grant DEC-2013/11/D/ST6/03073; However, large part of this work was done when M. Pilipczuk was affiliated with the University of Bergen and was supported by the aforementioned ERC grant n. 267959.

${ }^{\dagger}$ Department of Informatics, University of Bergen, Norway, pal.drange@ii.uib.no.

${ }^{\ddagger}$ Institute of Informatics, University of Warsaw, Poland, michal.pilipczuk@mimuw.edu.pl. 
characterized by a finite set of forbidden induced subgraphs: there is a finite list of graphs $H_{1}, H_{2}, \ldots, H_{p}$ such that any graph $G$ belongs to $\mathcal{G}$ if and only if $G$ does not contain any $H_{i}$ as an induced subgraph. Although many studied graph classes satisfy this property, there are important examples, like chordal or interval graphs, that are outside this regime.

For this reason, the parameterized analysis of modification problems for graph classes characterized by a finite set of forbidden induced subgraphs focused on studying the design of polynomial kernelization algorithms (polynomial kernels); Recall that such an algorithm is required, given an input instance $(G, k)$ of the problem, to preprocess it in polynomial time and obtain an equivalent output instance $\left(G^{\prime}, k^{\prime}\right)$, where $\left|G^{\prime}\right|, k^{\prime} \leq p(k)$ for some polynomial $p$. That is, the question is the following: can you, using polynomial-time preprocessing only, bound the size of the tackled instance by a polynomial function depending only on $k$ ?

For vertex deletion problems the answer is again quite simple: As long as $\mathcal{G}$ is characterized by a finite set of forbidden induced subgraphs, the task is to hit all the copies of these subgraphs (so-called obstacles) that are originally contained in the graph. Hence, one can construct a simple reduction to the $d$-Hitting SET problem for a constant $d$ depending on $\mathcal{G}$, and use the classic $O\left(k^{d}\right)$ kernel for the latter that is based on the sunflower lemma (see e.g. [11, 14]). For edge modifications problems, however, this approach fails utterly: every edge addition/deletion can create new obstacles, and thus it is not sufficient to hit only the original ones. For this reason, edge modification problems behave counterintuitively w.r.t. polynomial kernelization, and up to recently very little was known about their complexity.

On the positive side, kernelization of edge modification problems for well-studied graph classes was explored by Guo [19], who showed that four problems: Threshold Completion, Split Completion, Chain Completion, and Trivially Perfect Completion, all admit polynomial kernels. However, the study took a turn for the interesting when Kratch and Wahlström [23] showed that there is a graph $H$ on 7 vertices, such that the deletion problem to $H$-free graphs (the class of graphs not admitting $H$ as an induced subgraph) does not admit a polynomial kernel, unless the polynomial hierarchy collapses. This shows that the subtle differences between edge modification and vertex deletion problems have tremendous impact on the kernelization complexity.

Kratch and Wahlström conclude by asking whether there is a "simple" graph, like a path or a cycle, for which an edge modification problem does not admit a polynomial kernel under similar assumptions. The question was answered by Guillemot et al. [18] who showed that both for the class of $P_{\ell}$-free graphs (for $\ell \geq 7$ ) and for the class of $C_{\ell}$-free graphs (for $\ell \geq 4$ ), the edge deletion problems probably do not have polynomial kernelization algorithms. They simultaneously gave a cubic kernel for the COGRAPH EDITING problem, the problem of editing to a graph without induced paths on four vertices.

These results were later improved by Cai and Cai [6], who tried to obtain a complete dichotomy of the kernelization complexity of edge modification problems for classes of $H$-free graphs, for every graph $H$. The project has been almost fully successful - the question remains unresolved only for a finite number of graphs $H$. In particular, it turns out that the existence of a polynomial kernel for any of $H$-Free Editing, $H$-Free Edge Deletion, or $H$-Free Completion problems is in fact a very rare phenomenon, and basically happens only for specific, constant-size graphs $H$. In particular, for $H$ being a path or a cycle, the aforementioned three problems admit polynomial kernels if and only if $H$ has at most three edges.

At the same time, there is a growing interest in identifying parameterized problems that are solvable in subexponential parameterized time, i.e., in time $2^{o(k)} n^{O(1)}$. Although for many classic parameterized problems already known NP-hardness reductions show that the existence of such an algorithm would contradict the Exponential Time Hypothesis of Impagliazzo et al. [20], subexponential parameterized algorithms were known to exist for problems in restricted settings, like planar, or more generally $H$-minor free graphs [7], or tournaments [1]. See the book of Flum and Grohe [11] for a wider discussion.

Therefore, it was an immense surprise when Fomin and Villanger [15] showed that CHORDAL ComPLETION (also called Minimum FiLl-In) can be solved in time $2^{O(\sqrt{k} \log k)} n^{O(1)}$. Following this discovery, a new line of research was initiated. Ghosh et al. [17] showed that Split Completion is solvable in the same running time. Although Komusiewicz and Uhlmann [22] showed that we cannot expect CLusTER EDITING to be solvable in subexponential parameterized time, as shown by Fomin et al. [12], when the number of clusters in the target graph is sublinear in the number of allowed edits, this is possible nonetheless. 
Following these three positive examples, Drange et al. [9] showed that completion problems for trivially perfect graphs, threshold graphs and pseudosplit graphs all admit subexponential parameterized algorithms. Later, Bliznets et al. showed that both Proper Interval Completion and Interval COMPLETion also admit subexponential parameterized algorithms [3, 2].

Let us remark that in almost all these results, the known existence of a polynomial kernelization procedure for the problem played a vital role in designing the subexponential parameterized algorithm. Kernelization is namely used as an opening step that enables us to assume that the size of the considered graph is polynomial in the parameter $k$, something that turns out to be extremely useful in further reasonings. The only exception is the algorithm for the InTERval Completion problem [2], for which the existence of a polynomial kernel remains a notorious open problem. The need of circumventing this issue created severe difficulties in [2].

In this paper we study the TRIVIALly PERfECT EDITING problem. Recall that trivially perfect graphs are exactly graphs that do not contain a $P_{4}$ or a $C_{4}$ as an induced subgraph; see Section 2.2 for a structural characterization of this graph class. Interest in trivially perfect graphs started with the attempts to prove the strong perfect graph theorem. In recent times, new source of motivation has grown, with the realization that trivially perfect graphs are related to the width parameter treedepth (called also vertex ranking number, ordered chromatic number, and minimum elimination tree height). Although it had been known that both the completion and the deletion problem for trivially perfect graphs are NP-hard, it was open for a long time whether the editing version is NP-hard as well [4, 25].

This question was answered very recently by Nastos and Gao [27], who showed that the problem is indeed NP-hard. Actually, the work of Nastos and Gao focuses on exhibiting applications of trivially perfect graphs in social network theory, since this graph class may serve as a model for familial groups, communities in social networks showing a hierarchical nature. Specifically, the editing number to a trivially perfect graph ${ }^{1}$ can be used as a measure of how much a social network resembles a collection of hierarchies. Nastos and Gao also ask whether it is possibly to obtain a polynomial kernelization algorithm for this problem. The question about the existence of a polynomial kernel for TRIVIALLY PERFECT EDITING was then restated in a recent survey by Liu, Wang, and Guo [24], which nota bene contains a comprehensive overview of the current status of the research on the kernelization complexity of graph modification problems.

Our contribution. We answer the question of Nastos and Gao [27] and of Liu, Wang, and Guo [24] in affirmative by proving the following theorem.

Theorem 1. The problem Trivially Perfect Editing admits a proper kernel with $O\left(k^{7}\right)$ vertices.

Here, we say that a kernel (kernelization algorithm) is proper if it can only decrease the parameter, i.e., the output parameter $k^{\prime}$ satisfies $k^{\prime} \leq k$.

To prove Theorem 1, we employ an extensive analysis of the tackled instance, based on the equivalent structural definition of trivially perfect graphs. The main approach is to construct a small vertex modulator, a set of vertices whose removal results in obtaining a trivially perfect graph. However, since we are allowed only edge deletions and additions, this modulator just serves as a tool for exposing the structure of the instance. More specifically, we greedily pack disjoint obstructions into a set $X$, whose size can be guaranteed to be at most $4 k$, with the condition that to get rid of each of these obstructions, at least one edge must be edited inside the modulator per obstruction. Having obtained such a modulator, the rest of the graph, $G-X$, is trivially perfect, and we may apply the structural view on trivially perfect graphs to find irrelevant parts that can be reduced.

While the modulator technique is commonly used in kernelization, the new insight in this work is as follows. Since we work with an edge modification problem, we can be less restrictive about when an obstacle can be greedily packed into the modulator. For example, the obstacle does not need to be completely vertex-disjoint with the so far constructed $X$; sharing just one vertex is still allowed. This observation allows us to reason about the adjacency structure between $X$ and $V(G) \backslash X$, which is of great help when identifying irrelevant parts. We hope that this generic technique finds applications in other edge modification problems as well.

By slight modifications of our kernelization algorithm, we also obtain polynomial kernels for TRIVIALLY Perfect Deletion and Trivially Perfect Completion.

\footnotetext{
${ }^{1}$ Nastos and Gao use the terminology quasi-threshold graphs instead of trivially perfect graphs.
} 
Theorem 2. The problem Trivially PeRfeCt Deletion admits a proper kernel with $O\left(k^{7}\right)$ vertices.

Theorem 3. The problem TRIVIALly PeRfect Completion admits a proper kernel with $O\left(k^{7}\right)$ vertices.

To the best of our knowledge, no polynomial kernel for Trivially Perfect Deletion was known so far. For Trivially Perfect Completion, a cubic kernel was shown earlier by Guo [19]. Unfortunately, the work of Guo [19] is published only as a conference extended abstract, where it is only sketched how the approach yielding a quartic kernel for SPLIT DELETION could be used to obtain a cubic kernel for Trivially Perfect Completion. The details of this kernelization algorithm are deferred to the full version, which, alas, has not appeared. For this reason, we believe that our proof of Theorem 3 fills an important gap in the literature - the polynomial kernel for Trivially Perfect Completion is an important ingredient of the subexponential parameterized algorithm for this problem [9].

Finally, we show that Trivially Perfect Editing, in addition to being NP-complete, cannot admit a subexponential parameterized algorithm, provided that the Exponential Time Hypothesis holds.

Theorem 4. Under ETH, the TRIVIALly PERFECT EDITING problem is NP-hard and cannot be solved in time $2^{o(k)} n^{O(1)}$ or $2^{o(n+m)}$ even on graphs with maximum degree 4 .

In other words; the familial group measure cannot be computed in time subexponential in terms of the value of the measure. This stands in contrast with Trivially PERfECT Completion that admits a subexponential parameterized algorithm, and shows that TRIVIALLY PERFECT EDITING is more similar to Trivially Perfect Deletion, for which a similar lower bound has been proved earlier by Drange et al. [9]. In fact, our reduction can be used as an alternative proof of hardness of TRIVIALLY PERFECT DELETION as well.

Let us note that the NP-hardness reduction for Trivially Perfect Editing presented by Nastos and Gao [27] cannot be used to prove nonexistence of a subexponential parameterized algorithm, since it involves a cubic blow-up of the parameter (see Section 5 for details). To prove Theorem 4 , we resort to the technique used for similar hardness results by Komusiewicz and Uhlmann [22] and by Drange et al. [9].

\section{Preliminaries}

\subsection{Graphs and complexity}

Graphs. In this work we consider only undirected simple finite graphs. For a graph $G$, by $V(G)$ and $E(G)$ we denote the vertex and edge set of $G$, respectively. The size of a graph $G$ is defined as $|G|=|V(G)|+|E(G)|$.

For a vertex $v \in V(G)$, by $N_{G}(v)$ we denote the open neighborhood of $v$, i.e. $N_{G}(v)=\{u \in V(G) \mid$ $u v \in E(G)\}$. The closed neighborhood of $v$, denoted by $N_{G}[v]$, is defined as $N_{G}(v) \cup\{v\}$. These notions are extended to subsets of vertices as follows: $N_{G}[X]=\bigcup_{v \in X} N_{G}[v]$ and $N_{G}(X)=N_{G}[X] \backslash X$. We omit the subscript whenever $G$ is clear from context.

When $U \subseteq V(G)$ is a subset of vertices of $G$, we write $G[U]$ to denote the induced subgraph of $G$, i.e., the graph $G^{\prime}=\left(U, E_{U}\right)$ where $E_{U}$ is $E(G)$ restricted to $U$. The degree of a vertex $v \in V(G)$, denoted $\operatorname{deg}_{G}(v)$, is the number of vertices it is adjacent to, i.e., $\operatorname{deg}_{G}(v)=\left|N_{G}(v)\right|$. We denote by $\Delta(G)$ the maximum degree in the graph, i.e., $\Delta(G)=\max _{v \in V(G)} \operatorname{deg}(v)$. For a set $A$, we write $\left(\begin{array}{c}A \\ 2\end{array}\right)$ to denote the set of unordered pairs of elements of $A$; thus $E(G) \subseteq\left(\begin{array}{c}V(G) \\ 2\end{array}\right)$. By $\bar{G}$ we denote the complement of a graph $G$, i.e., $V(\bar{G})=V(G)$ and $E(\bar{G})=\left(\begin{array}{c}V(G) \\ 2\end{array}\right) \backslash E(G)$.

If $v$ and $u$ are such that $N[v]=N[u]$, then we call $v$ and $u$ true twins. Observe that $v$ and $u$ are adjacent if they are true twins. On the other hand, if $v$ and $u$ have $N(v)=N(u)$, then we call $v$ and $u$ false twins, and in this case we may observe that $v$ and $u$ are non-adjacent. If $X$ is an inclusion-wise maximal set of vertices such that for every pair of vertices $v$ and $u$ in $X$ they are true (resp. false) twins, then we call $X$ a true (resp. false) twin class.

For a graph $G$ and a set of vertices $X \subseteq V(G)$, we denote by $G-X$ the (induced subgraph) $G[V(G) \backslash X]$. When $F \subseteq\left(\begin{array}{c}V(G) \\ 2\end{array}\right)$, we write $G-F$ to denote the graph $G^{\prime}$ on vertex set $V(G)$ and edge set $E(G) \backslash F$. Finally, we let $G \triangle F$ be the graph on vertex set $V(G)$ and edge set $E(G) \triangle F$, where $\triangle$ denotes the symmetric difference; For two sets $A$ and $B, A \triangle B=(A \backslash B) \cup(B \backslash A)$. We will also say that two sets $A$ and $B$ are nested if $A \subseteq B$ or $B \subseteq A$. 
A vertex $v \in V(G)$ is universal if it is adjacent to all the other vertices of the graph. Note that the set of universal vertices of a graph forms a clique, which is also a true twin class. This clique will be denoted by uni $(G)$ and called the universal clique of $G$.

Modules and the modular decomposition. In our kernelization algorithm we will use the notion of a module in a graph.

Definition 2.1. Given a graph $G$, a set of vertices $M \subseteq V(G)$ is called a module if for any two vertices $v$ and $u$ in $M$, we have that $N(v) \backslash M=N(u) \backslash M$, i.e., all the vertices of $M$ have exactly the same neighborhood outside $M$.

Observe that for any graph $G$, any singleton $M=\{v\}$ is a module, and also $V(G)$ itself is a module. However, $G$ can contain a whole hierarchy of modules. This hierarchy can be captured using the following notion of a modular decomposition, introduced by Gallai [16]. The following description of a modular decomposition is taken verbatim from the work of Bliznets et al. [2] .

A module decomposition of a graph $G$ is a rooted tree $T$, where each node $t$ is labeled by a module $M^{t} \subseteq V(G)$, and is one of four types:

leaf: $t$ is a leaf of $T$, and $M^{t}$ is a singleton;

union: $G\left[M^{t}\right]$ is disconnected, and the children of $t$ are labeled with different connected components of $G\left[M^{t}\right]$

join: the complement of $G\left[M^{t}\right]$ is disconnected, and the children of $t$ are labeled with different connected components of the complement of $G\left[M^{t}\right]$

prime: neither of the above holds, and the children of $t$ are labeled with different modules of $G$ that are proper subsets of $M^{t}$, and are inclusion-wise maximal with this property.

Moreover, we require that the root of $T$ is labeled with the module $V(G)$. We need the following properties of the module decomposition.

Theorem 5 (see [26]). For a graph $G$, the following holds.

1. A module decomposition $\left(T,\left(M^{t}\right)_{t \in V(T)}\right)$ of $G$ exists, is unique, and computable in linear time.

2. At any prime node $t$ of $T$, the labels of the children form a partition of $M^{t}$. In particular, for each vertex $v$ of $G$ there exists exactly one leaf node with label $\{v\}$.

3. Each module $M$ of $G$ is either a label of some node of $T$, or there exists a union or join node $t$ such that $M$ is a union of labels of some children of $t$.

Let us remark that since in this work we do not optimize the running time of the kernelization algorithm, we do not need to compute the modular decomposition in linear time. Any simpler polynomial time algorithm would suffice (see the work of McConnell and Spinrad [26] for a literature overview).

Parameterized complexity. The running time of an algorithm is usually described as a function of the length of the input. To refine the complexity analysis of computationally hard problems, parameterized complexity introduced the notion of an extra "parameter" that is an additional part of a problem instance responsible for measuring its complexity. To simplify the notation, we will consider inputs to problems of the form $(G, k)$, which is a pair consisting of a graph $G$ and a nonnegative integer $k$. A problem is then said to be fixed parameter tractable if there is an algorithm which solves the problem in time $f(k) \cdot \operatorname{poly}(|G|)$, where $f$ is any function, and poly: $\mathbb{N} \rightarrow \mathbb{N}$ any polynomial function. In the case when $f(k)=2^{o(k)}$ we say that the algorithm is a subexponential parameterized algorithm. When a problem $\Pi \subseteq \mathcal{G} \times \mathbb{N}$ is fixed-parameter tractable, where $\mathcal{G}$ is the class of all graphs, we say that $\Pi$ belongs to the complexity class FPT. For a more rigorous introduction to parameterized complexity we refer to the books of Downey and Fellows [8] and of Flum and Grohe [11].

A kernelization algorithm (or kernel) is a polynomial-time algorithm for a parameterized problem $\Pi$ that takes as input a problem instance $(G, k)$ and returns an equivalent instance $\left(G^{\prime}, k^{\prime}\right)$, i.e. $(G, k) \in$ $\Pi \Leftrightarrow\left(G^{\prime}, k^{\prime}\right) \in \Pi$, where both $\left|G^{\prime}\right|$ and $k^{\prime}$ are bounded by $f(k)$ for some function $f$. We then say that $f$ 


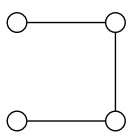

(a) $P_{4}$

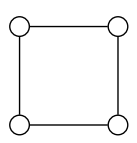

(b) $C_{4}$

Figure 1: Trivially perfect graphs are $\left\{C_{4}, P_{4}\right\}$-free.

is the size of the kernel. When $k^{\prime} \leq k$, we say that the kernel is a proper kernel. Specifically, a proper polynomial kernelization algorithm for $\Pi$ is a polynomial time algorithm which takes as input an instance $(G, k)$ and returns an equivalent instance $\left(G^{\prime}, k^{\prime}\right)$ with $k^{\prime} \leq k$ and $\left|G^{\prime}\right| \leq p(k)$ for some polynomial function $p$.

Tools for lower bounds. As evidence that TRIVIAlly PERfECT EDITING cannot be solved in subexponential parameterized time $2^{o(k)} n^{O(1)}$, we will use the Exponential Time Hypothesis, formulated by Impagliazzo, Paturi, and Zane [20].

Exponential Time Hypothesis (Exponential Time Hypothesis, ETH). There exists a positive real number $s$ such that 3 SAT with $n$ variables and $m$ clauses cannot be solved in time $2^{s n}(n+m)^{O(1)}$.

Impagliazzo, Paturi, and Zane [20] proved a fundamental result called Sparsification Lemma, which can serve as a Turing reduction from an arbitrary instance of 3SAT to an instance where the number of clauses is linear in the number of variables. Thus, the following statement is an immediate corollary of the Sparsification Lemma.

Proposition 2.2 ([20]). Unless ETH fails, there exists a positive real number $s$ such that 3SAT with $n$ variables and $m$ clauses cannot be solved in time $2^{s(n+m)}(n+m)^{O(1)}$. In particular, 3SAT does not admit an algorithm with time complexity $2^{o(n+m)}(n+m)^{O(1)}$.

\subsection{Trivially Perfect Graphs}

Combinatorial properties. A graph $G$ is trivially perfect if and only if it does not contain a $C_{4}$ or a $P_{4}$ as an induced subgraph. That is, trivially perfect graphs are defined by the forbidden induced subgraph family $F=\left\{C_{4}, P_{4}\right\}$ (see Figure 1). However, we mostly rely on the following recursive characterization of the trivially perfect graphs:

Proposition 2.3 ([21]). The class of trivially perfect graphs can be defined recursively as follows:

- $K_{1}$ is a trivially perfect graph.

- Adding a universal vertex to a trivially perfect graph results in a trivially perfect graph.

- The disjoint union of two trivially perfect graphs results in a trivially perfect graph.

Based on Proposition 2.3, a superset of the current authors [9] proposed the following notion of a decomposition for trivially perfect graphs. In the following, for a rooted tree $T$ and vertex $t \in V(T)$, by $T_{t}$ we denote the subtree of $T$ rooted at $t$.

Definition 2.4 (Universal clique decomposition, [9]). A universal clique decomposition $(U C D)$ of a connected graph $G$ is a pair $\mathcal{T}=\left(T=\left(V_{T}, E_{T}\right), \mathcal{B}=\left\{B_{t}\right\}_{t \in V_{T}}\right)$, where $T$ is a rooted tree and $\mathcal{B}$ is a partition of the vertex set $V(G)$ into disjoint nonempty subsets, such that

- if $v w \in E(G)$ and $v \in B_{t}, w \in B_{s}$, then either $t=s, t$ is an ancestor of $s$ in $T$, or $s$ is an ancestor of $t$ in $T$, and

- for every node $t \in V_{T}$, the set of vertices $B_{t}$ is the universal clique of the induced subgraph $G\left[\bigcup_{s \in V\left(T_{t}\right)} B_{s}\right]$

We call the vertices of $T$ nodes and the sets in $\mathcal{B}$ bags of the universal clique decomposition $(T, \mathcal{B})$. By slightly abusing notation, we often identify nodes with corresponding bags. Note that by the definition, 
in a universal clique decomposition every non-leaf node $t$ has at least two children, since otherwise the bag $B_{t}$ would not comprise all the universal vertices of the graph $G\left[\bigcup_{s \in V\left(T_{t}\right)} B_{s}\right]$.

The following lemma explains the connection between trivially perfect graphs and universal clique decompositions.

Lemma $2.5([9])$. A connected graph $G$ admits a universal clique decomposition if and only if it is trivially perfect. Moreover, such a decomposition is unique up to isomorphisms.

Note that a universal clique decomposition can trivially be found in polynomial time by repeatedly locating universal vertices and connected components. Moreover, we can extend the notion of a universal clique decomposition also to a disconnected trivially perfect graph $G$. In this case, the universal clique decomposition of $G$ becomes a rooted forest consisting of universal clique decompositions of the connected components of $G$. Since a graph is trivially perfect if and only if each of its connected component is, Lemma 2.5 can be easily generalized to the following statement: Every (possibly disconnected) graph $G$ is trivially perfect if and only if it admits a universal clique decomposition, where the decomposition has the shape of a rooted forest. Moreover, this decomposition is unique up to isomorphism.

The following definition of a quasi-ordering of vertices respecting the UCD will be helpful when arguing the correctness of the kernelization procedure.

Definition 2.6. Let $(T, \mathcal{B})$ be the universal clique decomposition of a trivially perfect graph $G$. We impose a quasi-ordering $\preceq$ on vertices of $G$ defined as follows. Suppose vertex $u$ belongs to bag $B_{t}$ and vertex $v$ belongs to bag $B_{s}$. Then $u \preceq v$ if and only if $t=s$ or $t$ is an ancestor of $s$ in the rooted forest $T$.

Thus, classes of vertices pairwise equivalent with respect to $\preceq$ are exactly formed by the bags of $\mathcal{B}$, and otherwise the ordering respects the rooted structure of $T$. Note that since the UCD of a trivially perfect graph is unique up to isomorphism, the quasi-ordering $\preceq$ is uniquely defined and can be computed in polynomial time.

Computational problems. In this work we are mainly interested in the TrIVIALLY PERFECT EDITING problem, defined formally as follows:

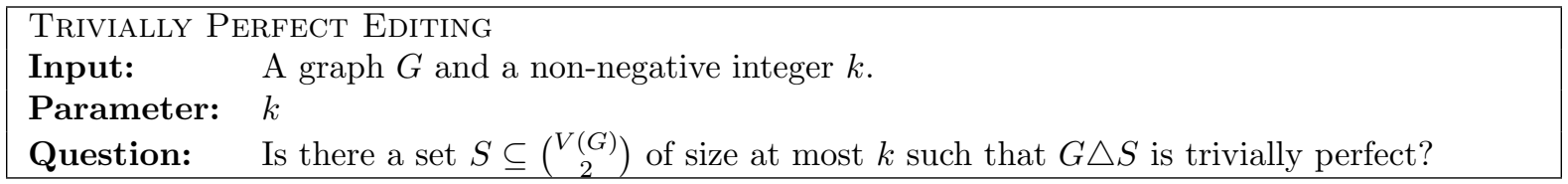

For a graph $G$, any set $F \subseteq\left(\begin{array}{c}V(G) \\ 2\end{array}\right)$ for which $G \triangle F$ is trivially perfect will henceforth be referred to as an editing set. An editing set is minimal if no proper subset $F^{\prime} \subsetneq F$ is also an editing set.

In the Trivially Perfect Deletion and Trivially Perfect Completion problems we allow only edge deletions and edge additions, respectively. More formally, we require that the editing set $S$ is contained in, or disjoint from $E(G)$, respectively. In Section 3 we prove Theorem 1, that is, we show that Trivially Perfect Editing admits a kernel with $O\left(k^{7}\right)$. Actually, the character of our data reduction rules will be very simple; The kernelization algorithm will start with instance $(G, k)$, and perform only the following operations:

- edit some $e \in\left(\begin{array}{c}V(G) \\ 2\end{array}\right)$, decrement the budget $k$ by 1 , and terminate the algorithm if $k$ becomes negative; or

- remove some vertex $u$ of $G$ and proceed with instance $(G-u, k)$.

Thus, the kernel will essentially be an induced subgraph of $G$, modulo performing some edits whose safeness and necessity can be deduced. In the proofs of correctness, we will never use any minimality argument that exchanges edge deletions for completions, or vice versa. Therefore, the whole approach can be applied almost verbatim to Trivially Perfect Deletion and Trivially Perfect Completion, yielding proofs for Theorems 2 and 3 after very minor modifications. We hope that the reader will be convinced about this after understanding all the arguments of Section 3. However, for the sake of completeness we, in Section 4, review the modifications of the argumentation of Section 3 that are necessary to prove Theorems 2 and 3 . 
TP-set systems. In the kernelization algorithm we will need the following auxiliary definition and result.

Definition 2.7 (TP-set system). A set system $\mathcal{F} \subseteq 2^{U}$ over a ground set $U$ is called a TP-set system if for every $X_{1}$ and $X_{2}$ in $\mathcal{F}$ with $x_{1} \in X_{1} \backslash X_{2}$ and $x_{2} \in X_{2} \backslash X_{1}$, there is no $Y \in \mathcal{F}$ with $\left\{x_{1}, x_{2}\right\} \subseteq Y$.

The following property bounds the size of a TP-set system, which we need later:

Lemma 2.8. Let $\mathcal{F}$ be a TP-set system over a finite ground set $U$. Then the cardinality of $\mathcal{F}$ is at most $|U|+1$.

Proof. We proceed by induction on $|U|$, with the claim being trivial when $U=\emptyset$. Suppose $\mathcal{F}$ is a TP-set system over a ground set $U$, and let $X$ be a member of $\mathcal{F}$ that has the minimum cardinality among the nonempty ones (if there is no such set, then $|\mathcal{F}| \leq 1$ and we are done). The first observation is that if $Y_{1}$ and $Y_{2}$ are two nonempty members of $\mathcal{F}$ that satisfy $Y_{1} \backslash X=Y_{2} \backslash X$ (possibly $Y_{1}=X$ or $\left.Y_{2}=X\right)$, then in fact $Y_{1}=Y_{2}$. Suppose otherwise that there exist two such nonempty sets $Y_{1}, Y_{2} \in \mathcal{F}$ with $Y_{1} \cap X \neq Y_{2} \cap X$; W.l.o.g., suppose that there exists an element $x_{1} \in Y_{1} \backslash Y_{2} \subseteq X$, and hence $x_{1} \in X \backslash Y_{2}$. Since $X$ is of minimum cardinality, we have that $|X| \leq\left|Y_{2}\right|$. As $X \nsubseteq Y_{2}$, we infer that there exists an element $x_{2} \in Y_{2} \backslash X=Y_{1} \backslash X$. Consider the pair $\left\{x_{1}, x_{2}\right\}$ and observe that (a) $x_{1} \in X \backslash Y_{2}$, (b) $x_{2} \in Y_{2} \backslash X$, and (c) $\left\{x_{1}, x_{2}\right\} \subseteq Y_{1}$. This contradicts the definition of a TP-set system.

Define a set system $\mathcal{F}^{\prime}$ over the ground set $U \backslash X$ as follows:

$$
\mathcal{F}^{\prime}=\{Y \backslash X: Y \in \mathcal{F}, Y \neq \emptyset\} .
$$

Clearly, $\mathcal{F}^{\prime}$ is a TP-set system over a strictly smaller ground set, so from the induction hypothesis we infer that $\left|\mathcal{F}^{\prime}\right| \leq|U \backslash X|+1$. Moreover, from the observation of the previous paragraph we infer that sets $Y \backslash X$ are pairwise different for $Y \in \mathcal{F}, Y \neq \emptyset$, and hence $|\mathcal{F}| \leq\left|\mathcal{F}^{\prime}\right|+1$ (the additive +1 comes from possibly having the empty set in $\mathcal{F}$ ). Concluding,

$$
|\mathcal{F}| \leq\left|\mathcal{F}^{\prime}\right|+1 \leq|U \backslash X|+1+1 \leq|U|-1+1+1=|U|+1
$$

\section{A Kernel for Trivially Perfect Editing}

This section is devoted to the proof of Theorem 1, stating that TRIVIALLY PERfECT EDITING admits a proper kernel with $O\left(k^{7}\right)$ vertices. As usual, the kernelization algorithm will be given as a sequence of data reduction rules: simple preprocessing procedures that, if applicable, simplify the instance at hand. For each rule we shall prove two results: (a) that applicability of the rule can be recognized in polynomial time, and (b) that the rule is safe, i.e., the resulting instance is equivalent to the input one. At the end of the proof we will argue that if no rule is applicable, then the size of the instance must be bounded by $O\left(k^{7}\right)$. Some rules will decrement the budget $k$ for edge edits; If this budget drops below zero, we may conclude that we are dealing with a no-instance, so we immediately terminate the algorithm and provide a constant-size trivial no-instance as the obtained kernel, for example the instance $\left(C_{4}, 0\right)$.

Before starting the formal description, let us give a brief overview of the structure of the proof. In Section 3.1 we give some preliminary basic rules, which mostly deal with situations where we can find a large number of induced $C_{4} \mathrm{~s}$ and $P_{4} \mathrm{~s}$ in the graph (henceforth called obstacles), which share only one edge or non-edge. We then infer that this edge or non-edge has to be included in any editing set of size at most $k$, and hence we can perform the necessary edit and decrement the budget.

In Section 3.2 we perform a greedy algorithm that iteratively packs disjoint induced $C_{4} \mathrm{~s}$ and $P_{4} \mathrm{~s}$ in the graph. Note that if we are able to pack more than $k$ of them, then this certifies that the considered instance does not have a solution, and we can terminate the algorithm. Hence, if $X$ is the union of vertex sets of the packed obstacles, then $|X| \leq 4 k$ and $G-X$ is a trivially perfect graph. Uncovering such a set $X$, which we call a TP-modulator, imposes a lot of structure on the considered instance, and is the key for further analysis of irrelevant parts of the input.

Although the applied modulator technique is standard in the area of kernelization for graph modification problems, in this paper we introduce a new twist to it that may have possible further applications. Namely, we observe that since we consider edge editing problems, the packed obstacles do not have to be entirely 


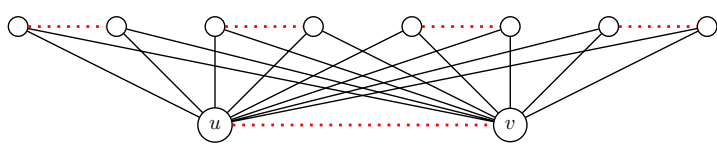

(a) Rule 1: There are four $C_{4} \mathrm{~S}$ sharing only the vertices $u$ and $v$. Unless the edge $u v$ is added, we must use at least as many edits as the size of the non-matching.

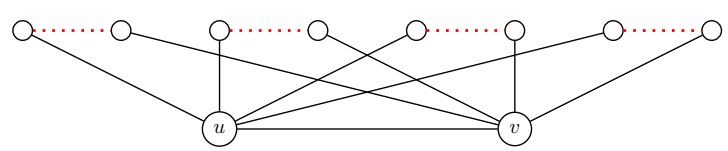

(b) Rule 2: There are four $P_{4} \mathrm{~S}$ sharing only the vertices $u$ and $v$. Unless the edge $u v$ is deleted, we must use at least as many edits as the size of the non-matching.

Figure 2: Illustrations of Rules 1 and 2. The red dotted edges are non-edges; They form a matching in the complement graph. In each of the cases, the only common vertices are $u$ and $v$.

vertex-disjoint, but the next obstacle can be packed even if it shares one vertex with the union of vertex sets of the previous obstacles; In some limited cases even having two vertices in common is permitted. Thus, the obtained modulator $X$ has the property that not only is there no obstacle in the graph $G$ that is vertex-disjoint with $X$, but even the existence of obstacles sharing one vertex with $X$ is forbidden. This simple observation enables us to reason about the adjacency structure between $X$ and $V(G) \backslash X$. In Section 3.3 we analyze this structure in order to prove the most important technical result of the proof: The number of subsets of $X$ that are neighborhoods within $X$ of vertices from $V(G) \backslash X$ is bounded polynomially in $k$; see Lemma 3.8 .

In Section 3.4 we proceed to analyze the trivially perfect graph $G-X$. Having the polynomial bound on the number of neighborhoods within $X$, we can locate in the UCD of $G-X$ a polynomial (in $k$ ) number of important bags, where something interesting from the point of view of $X$-neighborhoods happens. The parts between the important bags have very simple structure. They are either tassels: sets of trees hanging below some important bag, where each such tree is a module in the whole graph $G$; or combs: long paths stretched between two important bags where all the vertices of subtrees attached to the path have exactly the same neighborhood in $X$. Tassels and combs are treated differently: Large tassels contain large trivially perfect modules in $G$ that can be reduced quite easily, however for combs we need to devise a quite complicated irrelevant vertex rule that locates a vertex that can be safely discarded in a long comb. The module reduction rules are described in Section 3.5, while in Section 3.6 we reduce the sizes of tassels and combs and conclude the proof.

\subsection{Basic rules}

In this section we introduce the first two basic reduction rules. In the argumentation of the next sections, we will assume that none of these rules is applicable. An instance satisfying this property will be called reduced.

Rule 1. For an instance $(G, k)$ with $u v \notin E(G)$, if there is a matching of size at least $k+1$ in $\overline{G[N(u) \cap N(v)]}$, then add edge $u v$ to $G$ and decrease $k$ by one, i.e., return the new instance $(G+u v, k-1)$.

Rule 2. For an instance $(G, k)$ with $u v \in E(G)$ and $N_{1}=N(u) \backslash N[v]$ and $N_{2}=N(v) \backslash N[u]$, if there is a matching in $\bar{G}$ between $N_{1}$ and $N_{2}$ of size at least $k+1$, then delete edge $u v$ from $G$ and decrease $k$ by one, i.e., return the new instance $(G-u v, k-1)$.

Lemma 3.1. Applicability of Rules 1 and 2 can be recognized in polynomial time. Moreover, both these rules are safe, i.e., the input instance $(G, k)$ is a yes-instance if and only if the output instance $\left(G^{\prime}, k-1\right)$ is a yes-instance.

Proof. Observe that verifying applicability of Rule 1 or 2 to a fixed (non-)edge $u v$ boils down to computing the cardinality of the maximum matching in an auxiliary graph. This problem is well-known to be solvable in polynomial time [10]. Thus, by iterating over all edges and non-edges of $G$ we obtain polynomial time algorithms for recognizing applicability of Rules 1 and 2. We proceed to the proof of the safeness for both rules.

Rule 1: Let $x_{0} y_{0}, x_{1} y_{1}, \ldots, x_{k} y_{k}$ be edges of the found matching in $\overline{G[N(u) \cap N(v)]}$. Observe that for each $i, 0 \leq i \leq k$, vertices $u, x_{i}, v, y_{i}$ induce a $C_{4}$ in $G$. These induced $C_{4}$ s share only the non-edge $u v$, hence any editing set that does not contain $u v$ must contain at least one element of $\left(\begin{array}{c}\left\{u, x_{i}, v, y_{i}\right\} \\ 2\end{array}\right) \backslash\{u v\}$, 
and consequently be of size at least $k+1$. We infer that every editing set for $G$ that has size at most $k$ has to include the edge $u v$, and the safeness of the rule follows.

Rule 2: We proceed similarly as for Rule 1. Suppose $x_{0} y_{0}, x_{1} y_{1}, \ldots, x_{k} y_{k}$ is the found matching in $\bar{G}$, where $x_{i} \in N_{1}$ and $y_{i} \in N_{2}$ for $0 \leq i \leq k$. Then vertices $x_{i}, u, v, y_{i}$ induce a $P_{4}$, and all these $P_{4}$ s for $0 \leq i \leq k$ pairwise share only the edge $u v$. Similarly as for Rule 1 , we conclude that every editing set for $G$ of size at most $k$ has to contain $u v$, and the safeness of the rule follows.

We can now use Lemma 3.1 to apply Rules 1 and 2 exhaustively; note that each application reduces the budget $k$, hence at most $k$ applications can be performed before discarding the instance as a no-instance. From now on, we assume that the considered instance $(G, k)$ is reduced.

\subsection{Modulator construction}

We now move to the construction of a small modulator whose raison d'être is to expose structure in the considered graph $G$. We say that a subset $W \subseteq V(G)$ with $|W|=4$ is an obstruction if $G[W]$ is isomorphic to a $C_{4}$ or a $P_{4}$. Formally, our modulator will be compliant to the following definition.

Definition 3.2 (TP-modulator). Let $(G, k)$ be an instance of Trivially Perfect Editing. A subset $X \subseteq V(G)$ is a TP-modulator if for every obstruction $W$ the following holds (see Figure 3):

- $|W \cap X| \geq 2$, and

- if $|W \cap X|=2$, then it cannot happen that $G[W]$ is a $C_{4}$ of the form $x_{1}-y_{1}-y_{2}-x_{2}-x_{1}$ or a $P_{4}$ of the form $x_{1}-y_{1}-y_{2}-x_{2}$, where $W \cap X=\left\{x_{1}, x_{2}\right\}$.

We call a TP-modulator $X$ small if $|X| \leq 4 k$.

In particular, observe that for a TP-modulator $X$ there is no obstacle disjoint with $X$, so $G-X$ is trivially perfect. The following result shows that from now we can assume that a small TP-modulator is given to us.

Lemma 3.3. Given an instance $(G, k)$ for Trivially Perfect Editing, we can in polynomial time construct a small TP-modulator $X \subseteq V(G)$, or correctly conclude that $(G, k)$ is a no-instance.

Proof. The algorithm starts with $X_{0}=\emptyset$, and iteratively constructs an increasing family of sets $X_{0} \subseteq$ $X_{1} \subseteq X_{2} \subseteq \ldots$. In the $i$ th iteration we look for an obstacle $W$ that contradicts the fact that $X_{i-1}$ is a TP-modulator according to Definition 3.2, by verifying all the quadruples of vertices in $O\left(n^{4}\right)$ time. If this check verifies that $X_{i-1}$ is a TP-modulator, then we terminate the algorithm and output $X=X_{i-1}$. Otherwise, we set $X_{i}=X_{i-1} \cup W$ and proceed to the next iteration. Moreover, if we performed $k+1$ iterations, i.e., successfully constructed set $X_{k+1}$, then we terminate the algorithm concluding that $(G, k)$ is a no-instance. Since in each iteration the next $X_{i}$ grows by at most 4 vertices, we infer that if we succeed in outputting a TP-modulator $X$, then it has size at most $4 k$.

We are left with proving that if the algorithm successfully constructed $X_{k+1}$, then $(G, k)$ is a noinstance. To this end, we prove by induction on $i$ that for every $i=0,1, \ldots, k+1$ and every editing set $F$ for $G$, it holds that $\left|F \cap\left(\begin{array}{c}X_{i} \\ 2\end{array}\right)\right| \geq i$. Indeed, from this statement for $i=k+1$ we can infer that every editing set for $G$ has size at least $k+1$, so $(G, k)$ is a no-instance. The base of the induction is trivial, so for the induction step suppose that $X_{i}=X_{i-1} \cup W$, where $W$ is an obstacle with $\left|W \cap X_{i-1}\right| \leq 1$ or having the form described in the second point of Definition 3.2.

First, if $\left|W \cap X_{i-1}\right| \leq 1$, then $\left(\begin{array}{c}W \\ 2\end{array}\right)$ is disjoint with $\left(\begin{array}{c}X_{i-1} \\ 2\end{array}\right)$. Since $F$ is an editing set for $G$, we have that $F \cap\left(\begin{array}{c}W \\ 2\end{array}\right) \neq \emptyset$, and hence

$$
\left|F \cap\left(\begin{array}{c}
X_{i} \\
2
\end{array}\right)\right| \geq\left|F \cap\left(\begin{array}{c}
X_{i-1} \\
2
\end{array}\right)\right|+\left|F \cap\left(\begin{array}{c}
W \\
2
\end{array}\right)\right| \geq i-1+1=i,
$$

by the induction hypothesis. Second, if $\left|W \cap X_{i-1}\right|=2$ and $W$ has one of the two forms described in the second point of Definition 3.2, then it is easy to see that $F$ in fact has to have a nonempty intersection with $\left(\begin{array}{c}W \\ 2\end{array}\right) \backslash\left\{x_{1} x_{2}\right\}$ : editing only the (non)edge $x_{1} x_{2}$ would turn a $C_{4}$ into a $P_{4}$ or vice versa. Since $\left(\begin{array}{c}W \\ 2\end{array}\right) \backslash\left\{x_{1} x_{2}\right\}$ is disjoint with $\left(\begin{array}{c}X_{i-1} \\ 2\end{array}\right)$, we analogously obtain that

$$
\left|F \cap\left(\begin{array}{c}
X_{i} \\
2
\end{array}\right)\right| \geq\left|F \cap\left(\begin{array}{c}
X_{i-1} \\
2
\end{array}\right)\right|+\left|F \cap\left(\left(\begin{array}{c}
W \\
2
\end{array}\right) \backslash\left\{x_{1} x_{2}\right\}\right)\right| \geq i-1+1=i .
$$




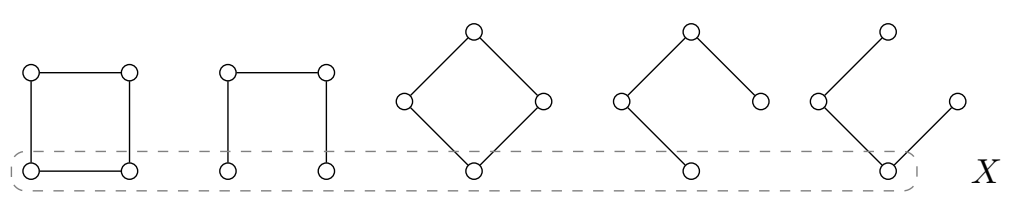

Figure 3: Forbidden patterns of intersection between an obstruction and a TP-modulator $X$.

By applying Lemma 3.3, from now on we assume that we are given a small TP-modulator $X$ in $G$.

\subsection{Bounding the number of neighborhoods in a TP-modulator}

Recall that we exposed a small TP-modulator $X$ in the input graph $G$. In polynomial time we compute the universal clique decomposition $\mathcal{T}=(T, \mathcal{B})$ of the trivially perfect graph $G-X$. The goal of this section is to analyze the structure of neighborhoods within $X$ of vertices residing outside $X$.

Definition 3.4 ( $X$-neighborhood). Let $G$ be a graph and $X \subseteq V(G)$. For a vertex $v \in V(G) \backslash X$, the $X$-neighborhood of $v$, denoted $N_{G}^{X}(v)$, is the set $N_{G}(v) \cap X$. The family of $X$-neighborhoods of $G$ is the set $\left\{N_{G}^{X}(v): v \in V(G) \backslash X\right\}$.

Again, we shall omit the subscript $G$ whenever this does not lead to any confusion. Recall that the UCD $\mathcal{T}$ gives us a quasi-ordering $\preceq$ on the vertices of $G-X$. We have $u \preceq v$ if the bag to which $v$ belong is a descendant of the bag which $u$ belongs to, where every bag is considered its own descendant. We shall use the notation $u \prec v$ to denote that $u \preceq v$ and $v \npreceq u$. The following two lemmas show that the quasi-ordering $\preceq$ is compatible with the inclusion ordering of $X$-neighborhoods.

Lemma 3.5. If $u \prec v$ then $N^{X}(u) \supseteq N^{X}(v)$.

Proof. Suppose $u \in B_{t}$ and $v \in B_{s}$, where $t \neq s$ and $t$ is an ancestor of $s$ in the forest $T$. Recall that in a $\mathrm{UCD}$, every non-leaf node has at least two children, which means that there exists some node $s^{\prime}$ that is a descendant of $t$, but which is incomparable with $s$. Let $w$ be any vertex of $B_{s^{\prime}}$. From the definition of a $\mathrm{UCD}$ it follows that $u v, u w \in E(G)$ but $v w \notin E(G)$.

For the sake of contradiction suppose that $N^{X}(u) \nsupseteq N^{X}(v)$, which means there exists a vertex $x \in X$ with $x v \in E(G)$ and $x u \notin E(G)$. It follows that $\{x, u, v, w\}$ is an obstacle regardless of whether $w x$ is an edge or a non-edge: it is an induced $C_{4}$ if $w x \in E(G)$ and an induced $P_{4}$ if $w x \notin E(G)$. Thus we have uncovered an obstacle sharing only one vertex with $X$, contradicting the fact that $X$ is a TP-modulator.

Lemma 3.6. If $u, v \in B_{t}$ for some $B_{t} \in \mathcal{B}$, then $N^{X}(u) \subseteq N^{X}(v)$ or $N^{X}(v) \subseteq N^{X}(u)$.

Proof. Since $u, v \in B_{t}$, we have that $u v \in E(G)$. For the sake of contradiction, suppose that there exist some $x_{u} \in N^{X}(u) \backslash N^{X}(v)$ and $x_{v} \in N^{X}(v) \backslash N^{X}(u)$. It can be now easily seen that regardless whether $x_{u} x_{v}$ belongs to $E(G)$ or not, the quadruple $\left\{u, v, x_{u}, x_{v}\right\}$ forms one of the obstacles forbidden in the second point of the Definition 3.2. This is a contradiction with the fact that $X$ is a TP-modulator.

Lemmas 3.5 and 3.6 motivate the following refinement of the quasi-ordering $\preceq$ : If $u, v$ belong to different bags of $\mathcal{T}$, then we put $u \preceq_{N} v$ if and only if $u \preceq v$, and if they are in the same bag, then $u \preceq_{N} v$ if and only if $N^{X}(u) \supseteq N^{X}(v)$. Thus, by Lemma $3.6 \preceq_{N}$ refines $\preceq$ by possibly splitting every bag of $\mathcal{T}$ into a family of linearly ordered equivalence classes. Moreover, by Lemmas 3.5 and 3.6 we have the following corollary.

Corollary 3.7. If $u \preceq_{N} v$ then $N^{X}(u) \supseteq N^{X}(v)$.

Observe that for a pair of vertices $u, v \in V(G) \backslash X$, the following conditions are equivalent: (a) $u$ and $v$ are comparable w.r.t $\preceq$, (b) $u$ and $v$ are comparable w.r.t. $\preceq_{N}$, and (c) $u v \in E(G)$. We have now prepared all the tools needed to prove the main lemma from this section. 
Lemma 3.8. If $(G, k)$ is a reduced instance for Trivially Perfect Editing and $X$ is a small $T P$-modulator, then the number of different $X$-neighborhoods is at most $O\left(k^{4}\right)$.

Proof. Let $\mathcal{F}$ be the family of $X$-neighborhoods in $G$. For every $Z \in \mathcal{F}$, let us choose an arbitrary vertex $v_{Z} \in V(G) \backslash X$ with $Z=N^{X}\left(v_{Z}\right)$. We split $\mathcal{F}$ into two subfamilies: The first family $\mathcal{F}_{1}$ contains all the sets of $\mathcal{F}$ that contain the endpoints of some non-edge in $G[X]$, whereas the second family $\mathcal{F}_{2}$ contains all the sets of $\mathcal{F}$ that induce complete graphs in $G[X]$. We bound the sizes of $\mathcal{F}_{1}$ and $\mathcal{F}_{2}$ separately.

Bounding $\left|\mathcal{F}_{1}\right|$ : Let $x y$ be a non-edge of $G[X]$, and for $2 \leq \kappa \leq|X|$ let $\mathcal{F}_{1}^{x y, \kappa}$ be the family of those sets of $\mathcal{F}_{1}$ that contain $\{x, y\}$ and have cardinality exactly $\kappa$. Take any distinct $Z_{1}, Z_{2} \in \mathcal{F}_{1}^{x y, \kappa}$, and observe that they are not nested since both have size $\kappa$. By Corollary 3.7, this means that vertices $v_{Z_{1}}$ and $v_{Z_{2}}$ are incomparable w.r.t. $\preceq_{N}$, so $v_{Z_{1}} v_{Z_{2}} \notin E(G)$. Hence, set $\left\{v_{Z}: Z \in \mathcal{F}_{1}^{x y, \kappa}\right\}$ is independent in $G$. Observe now that if we had that $\left|\left\{v_{Z}: Z \in \mathcal{F}_{1}^{x y, \kappa}\right\}\right| \geq 2 k+2$, then Rule 1 would be applicable to the non-edge $x y$. Since we assume that the instance is reduced, we conclude that $\left|\left\{v_{Z}: Z \in \mathcal{F}_{1}^{x y, \kappa}\right\}\right| \leq 2 k+1$, and hence also $\left|\mathcal{F}_{1}^{x y, \kappa}\right| \leq 2 k+1$. By summing through all the $\kappa$ between 2 and $|X|$ and through all the non-edges of $G[X]$, we infer that

$$
\left|\mathcal{F}_{1}\right| \leq\left(\begin{array}{c}
4 k \\
2
\end{array}\right) \cdot 4 k \cdot(2 k+1)=O\left(k^{4}\right)
$$

Bounding $\left|\mathcal{F}_{2}\right|$ : Consider any pair of $X$-neighborhoods $Z_{1}, Z_{2} \in \mathcal{F}_{2}$ such that they are not nested, and moreover there exist vertices $x_{1} \in Z_{1} \backslash Z_{2}$ and $x_{2} \in Z_{2} \backslash Z_{1}$ such that $x_{1} x_{2} \in E(G)$. Since $Z_{1}$ and $Z_{2}$ are not nested, by Corollary 3.7 we infer that $v_{Z_{1}}$ and $v_{Z_{2}}$ are incomparable w.r.t. $\preceq_{N}$, and hence $v_{Z_{1}} v_{Z_{2}} \notin E(G)$. Observe that then $G\left[\left\{v_{Z_{1}}, v_{Z_{2}}, x_{1}, x_{2}\right\}\right]$ is an induced $P_{4}$; however, the existence of such an obstacle is not forbidden by the definition of a TP-modulator.

Create an auxiliary graph $H$ with $V(H)=\mathcal{F}_{2}$, and put $Z_{1} Z_{2} \in E(H)$ if and only if $Z_{1}$ and $Z_{2}$ satisfy the condition from the previous paragraph, i.e., $Z_{1}$ and $Z_{2}$ are not nested and there exist $x_{1} \in Z_{1} \backslash Z_{2}$ and $x_{2} \in Z_{2} \backslash Z_{1}$ with $x_{1} x_{2} \in E(G)$. Run the classic greedy 2-approximation algorithm for vertex cover in $H$. This algorithm either finds a matching $M$ in $H$ of size more than $\left(\begin{array}{c}4 k \\ 2\end{array}\right) \cdot k$, or a vertex cover $C$ of $H$ of size at most $2 \cdot\left(\begin{array}{c}4 k \\ 2\end{array}\right) \cdot k$. In the first case, assign each edge $Z_{1} Z_{2}$ of $M$ to the corresponding edge $x_{1} x_{2}$ of $G[X]$ as in the definition of the edges of $H$. Observe that since $|X| \leq 4 k$, then some edge $x_{1} x_{2} \in G[X]$ is assigned at least $k+1$ times. Then it is easy to see that the sets $\left\{v_{Z_{1}}, v_{Z_{2}}, x_{1}, x_{2}\right\}$ for $Z_{1} Z_{2}$ being edges of $M$ assigned to $x_{1} x_{2}$ induce $P_{4}$ s that share only the edge $x_{1} x_{2}$, and hence Rule 2 would be applicable to $x_{1} x_{2}$. This is a contradiction with the assumption that $(G, k)$ is reduced. Hence, we can assume that we have successfully constructed a vertex cover $C$ of $H$ of size at most $2 \cdot\left(\begin{array}{c}4 k \\ 2\end{array}\right) \cdot k=O\left(k^{3}\right)$.

Let now $\mathcal{F}_{2}^{\prime}=\mathcal{F}_{2} \backslash C$. Since $\mathcal{F}_{2}^{\prime}$ is independent in $H$, it follows that for any non-nested $Z_{1}, Z_{2} \in \mathcal{F}_{2}^{\prime}$ and any $x_{1} \in Z_{1} \backslash Z_{2}, x_{2} \in Z_{2} \backslash Z_{1}$, we have that $x_{1} x_{2} \notin E(G)$. Since the sets of $\mathcal{F}_{2}^{\prime}$ induce complete graphs in $G[X]$, this means that in particular there is no set $Z_{3} \in \mathcal{F}_{2}^{\prime}$ that contains both $x_{1}$ and $x_{2}$. This proves that the family $\mathcal{F}_{2}^{\prime}$ is a TP-set system with $X$ as ground set, so by Lemma 2.8 we infer that $\left|\mathcal{F}_{2}^{\prime}\right| \leq|X|+1 \leq 4 k+1$. Concluding,

$$
\left|\mathcal{F}_{2}\right| \leq|C|+\left|\mathcal{F}_{2}^{\prime}\right| \leq O\left(k^{3}\right)+4 k+1=O\left(k^{3}\right),
$$

and $|\mathcal{F}| \leq\left|\mathcal{F}_{1}\right|+\left|\mathcal{F}_{2}\right|=O\left(k^{4}\right)+O\left(k^{3}\right)=O\left(k^{4}\right)$.

\subsection{Locating important bags}

In the previous section we analyzed the structure of neighborhoods that nodes from $V(G) \backslash X$ have in $X$. Our goal in this section is to perform the symmetric analysis: to understand, how the neighborhood of a fixed $x \in X$ in $V(G) \backslash X$ looks like. Eventually, we aim to locate a family $I$ of $O(k)$ important bags, where some non-trivial behavior w.r.t. the neighborhoods of vertices of $X$ happens. Then, we will perform a lowest common ancestor-closure on the set $I$, thus increasing its size to at most twice. After performing this step, all the connected components of $T-I$ have very simple structure from the point of view of their neighborhoods in $X$. As there are only $O(k)$ such components, we will be able to kernelize them separately.

The following definition and lemma explains what are the types of neighborhoods that vertices of $X$ can have in $V(G) \backslash X$. To simplify the notation, in the following we treat $\preceq$ also as a partial order on the 


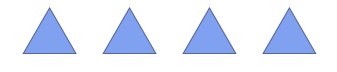

(a) Type 0: $x$ sees a disjoint union of connected components.

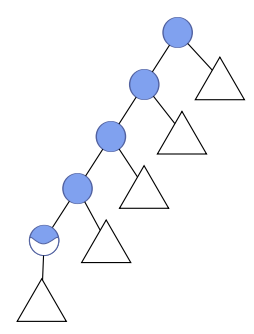

(b) Type 1: $x$ sees all the vertices in bags from a root and to a point in a bag, and nothing else.

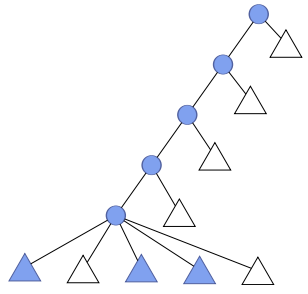

(c) Type 2: $x$ has as neighbors all the vertices from a root and down to a bag, and a collection of subtrees below that bag.

Figure 4: Three types of neighborhoods; simply denoted Type 0 , Type 1 , and Type 2 . The blue parts mark the possible neighborhoods of a vertex $x \in X$.

vertices of the forest $T$ denoting the ancestor-descendant relation, i.e., $s \preceq t$ if and only if $s$ is an ancestor of $t$ (possibly $s=t$ ).

Definition 3.9 (Type 0,1 , and 2 neighborhoods). Let $x \in X$ be any vertex and consider $U_{x}=N(x) \backslash X$. We say that $U_{x}$ is (see Figure 4):

- A neighborhood of Type 0 if $U_{x}$ is the union of the vertex sets of a collection of connected components of $G-X$.

- A neighborhood of Type 1 if there exists a node $t_{x} \in V(T)$ such that $\bigcup_{s \prec t_{x}} B_{s} \subseteq U_{x} \subseteq \bigcup_{s \preceq t_{x}} B_{s}$. In other words, $U_{x}$ consists of all the vertices contained in bags on the path from $t_{x}$ to the root of its subtree in $T$, where some vertices of $B_{t_{x}}$ itself may be excluded.

- A neighborhood of Type 2 if there exists a node $t_{x} \in V(T)$ and a collection $\mathcal{L}_{x}$ of subtrees of $T$ rooted at children of $t_{x}$ such that $U_{x}=\bigcup_{s \prec t_{x}} B_{s} \cup \bigcup_{S \in \mathcal{L}_{x}} \bigcup_{s \in V(S)} B_{s}$. In other words, $U_{x}$ is formed by all the vertices contained in bags on the path from $t_{x}$ to the root of its subtree in $T$, plus a selection of subtrees rooted in the children of $t_{x}$, where the vertices appearing in the bags of each such subtree are either all included in $U_{x}$ or all excluded from $U_{x}$.

Lemma 3.10. Let $x \in X$ be any vertex and consider $U_{x}=N(x) \backslash X$. Then $U_{x}$ is of Type 0, 1 or 2.

Proof. From Corollary 3.7 we infer that $U_{x}$ is closed downwards w.r.t. the quasi-ordering $\preceq_{N}$, i.e., if $v \in U_{x}$ and $u \preceq_{N} v$, then also $u \in U_{x}$. Let $S_{x}$ be the set of nodes of $T$ whose bags contain at least one vertex of $U_{x}$. It follows that $S_{x}$ is closed under taking ancestors in forest $T$. Moreover if $t \in S_{x}$, then the bags of all the ancestors of $t$ other than $t$ are fully contained in $U_{x}$.

Claim 3.11. Suppose $t, t^{\prime} \in S_{x}$ are two nodes that are incomparable w.r.t. $\preceq$. Then $U_{x} \supseteq \bigcup_{s \succeq t} B_{s}$ and $U_{x} \supseteq \bigcup_{s \succeq t^{\prime}} B_{s}$, i.e., $U_{x}$ contains all the vertices of all the bags contained in the subtrees of $T$ rooted at $t$ and $t^{\prime}$.

Proof. We prove the statement for the subtree rooted at $t^{\prime}$; The proof for the subtree rooted at $t$ is symmetric. Let $y$ and $y^{\prime}$ be arbitrary vertices of $B_{t} \cap U_{x}$ and $B_{t^{\prime}} \cap U_{x}$, respectively. For the sake of contradiction suppose there exists some $v \in \bigcup_{s \succ t^{\prime}} B_{s}$ such that $v x \notin E(G)$. Since $v \in \bigcup_{s \succ t^{\prime}} B_{s}$ and $t, t^{\prime}$ are incomparable w.r.t. $\preceq$, by the properties of the universal clique decomposition we have that $y y^{\prime} \notin E(G), v y \notin E(G)$ and $v y^{\prime} \in E(G)$. Since $x y, x y^{\prime} \in E(G)$ by the definition of $U_{x}$, we conclude that $\left\{y, y^{\prime}, x, v\right\}$ would induce a $P_{4}$ in $G$ that has only one vertex in common with $X$ (see Figure 5), a contradiction to the definition of a TP-modulator.

We now use Claim 3.11 to perform a case study that recognizes $U_{x}$ as a neighborhood of Type 0,1 , or 2 .

Suppose first that $U_{x}$ contains vertices of at least two distinct connected components of $G-X$. Let $C_{1}, C_{2}$ be any two such components, and let $T_{1}$ and $T_{2}$ be the trees of the forest $T$ that are UCDs 


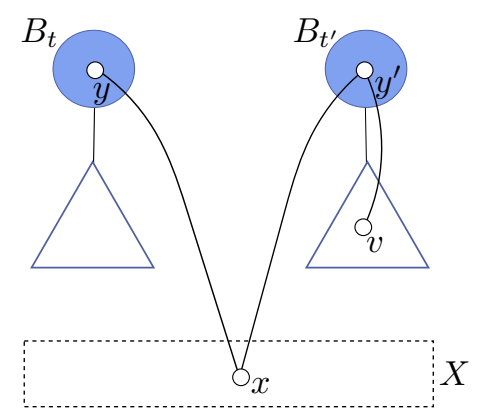

Figure 5: An induced $P_{4}, y x y^{\prime} v$, with only one vertex $x$ in the modulator, appearing in the proof of Claim 3.11.

of $C_{1}$ and $C_{2}$, respectively. Since $S_{x}$ is closed under taking ancestors in $T$, it follows that the roots of $T_{1}$ and $T_{2}$ belong to $S_{x}$. Claim 3.11 implies then that the entire vertex sets of $C_{1}$ and $C_{2}$ are contained in $U_{x}$. Since $C_{1}, C_{2}$ was an arbitrary pair of components containing a vertex of $U_{x}$, it follows that $U_{x}$ must be the union of vertex sets of a selection of connected components of $G-X$, i.e., a neighborhood of Type 0 .

Since $U_{x}=\emptyset$ is also a neighborhood of Type 0 , we are left with analyzing the case when $U_{x} \subseteq V\left(C_{0}\right)$ for $C_{0}$ being a connected component of $G-X$; Let $T_{0}$ be the UCD of $C_{0}$. Observe that if $U_{x}$ does not contain any pair of vertices incomparable w.r.t. $\preceq$, then $S_{x}$ must form a path from some node of $T_{0}$ to the root of $T_{0}$, and hence $U_{x}$ is a neighborhood of Type 1. Otherwise, there exists some node of $S_{x}$ such that at least two subtrees rooted at its children contain nodes from $S_{x}$. Let $t_{x}$ be such a node that is highest in $T_{0}$, and let $\mathcal{L}_{x}$ be the family of subtrees rooted at children of $t_{x}$ that contain nodes of $S_{x}$. Again applying Claim 3.11, we infer that $U_{x}$ contains all the vertices of all the bags of every subtree of $\mathcal{L}_{x}$ : for any two distinct subtrees $T_{1}, T_{2} \in \mathcal{L}_{x}, S_{x}$ contains the roots of $T_{1}$ and $T_{2}$, and hence by Claim $3.11 U_{x}$ contains all the vertices of all the bags of $T_{1}$ and $T_{2}$. Since $t_{x}$ was chosen to be the highest, it follows that $U_{x}$ is a neighborhood of Type 2 for node $t_{x}$ and selection of subtrees $\mathcal{L}_{x}$.

Clearly, for every $x \in X$ we can in polynomial time analyze $U_{x}$ and recognize it as a neighborhood of Type 0,1 , or 2 . Let $I_{0}$ be the set of nodes $t_{x}$ for vertices $x \in X$ for which $U_{x}$ is of Type 1 or 2 . To simplify the structure of $T-I_{0}$, we perform the lowest common ancestor-closure operation on $I_{0}$. The following variant of this operation is taken verbatim from the work of Fomin et al. [13].

Definition 3.12 ([13]). For a rooted tree $T$ and vertex set $M \subseteq V(T)$ the lowest common ancestor-closure (LCA-closure) is obtained by the following process. Initially, set $M^{\prime}=M$. Then, as long as there are vertices $x$ and $y$ in $M^{\prime}$ whose least common ancestor $w$ is not in $M^{\prime}$, add $w$ to $M^{\prime}$. When the process terminates, output $M^{\prime}$ as the LCA-closure of $M$. The following folklore lemma summarizes two basic properties of LCA-closures.

Lemma 3.13 ([13]). Let $T$ be a tree, $M \subseteq V(T)$ and $M^{\prime}=$ LCA-closure $(M)$. Then $\left|M^{\prime}\right| \leq 2|M|$ and for every connected component $C$ of $T-\bar{M}^{\prime},|N(C)| \leq 2$.

Construct now the set $I$ by taking LCA-closure $\left(I_{0}\right)$ and adding the root of every connected component of $T$ that contains a bag of $I_{0}$ (provided it is not already included). The nodes from $I$ will be called important nodes, or important bags. From Lemma 3.13 it follows that $|I| \leq 3|X| \leq 12 k$, and by the construction we infer that every connected component $C$ of $T-I$ is of one of the following three forms:

- $C$ is not adjacent to any node of $I$, and is thus simply a connected component of $T$ that does not contain any important bag.

- $C$ is adjacent to one node $a$ of $I$, and it is a subtree rooted at a child of $a$.

- $C$ is adjacent to two nodes $a$ and $b$ of $I$ such that $a$ is an ancestor of $b$. Then $C$ is formed by the internal nodes of the $a-b$ path in $T$, plus all the subtrees rooted at the other children of these internal nodes. 


\subsection{Module reduction}

In this section we give two new reduction rules: a twin reduction and a module reduction rule. These rules are executed exhaustively by the algorithm as Rules 3 and 4 . The reason why we introduce them now is that only after understanding the structural results of Sections 3.3 and 3.4, the motivation of these rules becomes apparent. Namely, these rules will be our main tools in reducing the sizes of parts of $G-X$ located between the important bags.

\subsubsection{Twin reduction}

Rule 3. If $T \subseteq V(G)$ is a true twin class of size $|T|>2 k+5$, and $v \in T$ is an arbitrarily picked vertex, then remove $v$ from the graph, i.e., proceed with the instance $(G-v, k)$.

Lemma 3.14. Applicability of Rule 3 can be recognized in polynomial time. Moreover, Rule 3 is safe, i.e., $(G, k)$ is a yes-instance if and only if $(G-v, k)$ is a yes-instance.

Proof. In order to recognize the applicability of Rule 3 we only need to inspect every true twin classes in the graph, which clearly can be done in polynomial time. We proceed to the proof of the safeness of the rule.

Let $T$ be a true twin class of size at least $2 k+5$ and let $v$ be the vertex the rule deleted. Since the class of trivially perfect graphs is hereditary, if $(G, k)$ is a yes-instance, it follows that $(G-v, k)$ is a yes-instance. Suppose now that $(G-v, k)$ is a yes-instance. Let $F$ be a set of edges with $|F| \leq k$ such that $(G-v) \triangle F$ is trivially perfect. We now show that $G \triangle F$ is also trivially perfect, which means that $F$ is also a solution to $(G, k)$. For the sake of contradiction, suppose $W$ is an obstruction in $G \triangle F$. Since $(G-v) \triangle F$ is trivially perfect, $W$ must contain the deleted vertex $v$. Since $F$ has size at most $k$, at most $2 k$ vertices of $T$ can be incident to an edge of $F$. Let $v_{1}, v_{2}, v_{3}$, and $v_{4}$ be four vertices of $T$ that are different from $v$ and are not incident to $F$. Then one of them, say $v_{1}$, is not contained in $W$. Since $v$ and $v_{1}$ are true twins both in $G$ and in $G \triangle F$, we can replace $v$ with $v_{1}$ in $W$ yielding a new set $W^{\prime}$ which is an obstruction in $G \triangle F$. However, since $v$ is not a member of $W^{\prime}$, we have that $W^{\prime}$ is an obstruction in $(G-v) \triangle F$, contradicting the assumption that $(G-v) \triangle F$ was trivially perfect.

\subsubsection{Module reduction}

Recall that a module is a set of vertices $M$ such that for every vertex $v$ in $V(G) \backslash M$, either $M \subseteq N(v)$ or $M \cap N(v)=\emptyset$; see Definition 2.1. The following rule enables us to reduce large trivially perfect modules.

Rule 4. Suppose $M \subseteq V(G)$ is a module such that $G[M]$ is trivially perfect and it contains an independent set of size at least $2 k+5$. Then let us take any independent set $I \subseteq M$ of size $2 k+4$, and we delete every vertex of $M$ apart from $I$, i.e., proceed with the instance $(G-(M \backslash I), k)$.

Observe that Rule 4 always deletes at least one vertex, since $|M| \geq 2 k+5$ and $|I|=2 k+4$. Actually, we could define a stronger rule where we only assume that $|M| \geq 2 k+5$; however, the current statement will be helpful in recognizing the applicability of Rule 4.

We first prove that the rule is indeed safe.

Lemma 3.15. Provided that $(G, k)$ is a reduced instance (w.r.t. Rules 1 and 2), then Rule 4 is safe, i.e., $(G, k)$ is a yes-instance if and only if $(G-(M \backslash I), k)$ is a yes-instance.

Proof. Let $A=M \backslash I$, and $G^{\prime}=G-A$. Since $G^{\prime}$ is an induced subgraph of $G$, by heredity, if $(G, k)$ is a yes-instance, then $\left(G^{\prime}, k\right)$ is a yes-instance. We proceed to the proof of the other direction. Suppose then that $\left(G^{\prime}, k\right)$ is a yes-instance, and let $F,|F| \leq k$, be a minimum-size editing set for $G^{\prime}$.

Claim 3.16. No vertex of $I$ is incident to any edit of $F$.

Proof. Since $F$ has minimum possible size, it is inclusion-wise minimal. We show that if $F_{I} \subseteq F$ is the set of edges of $F$ incident to a vertex of $I$ and $F^{\prime}=F \backslash F_{I}$, then $G^{\prime} \triangle F$ being trivially perfect implies $G^{\prime} \triangle F^{\prime}$ being trivially perfect. Since $|I|=2 k+4$, we can find at least four vertices $v_{1}, \ldots, v_{4} \in I$ that are not incident to any edit of $F$. Suppose that $G^{\prime} \triangle F^{\prime}$ is not trivially perfect. Then there is an obstruction $W$ in $G^{\prime} \triangle F^{\prime}$ containing at least one of the vertices of $I$ incident to an edge of $F$. Create $W^{\prime}$ by replacing every vertex of $(W \cap I) \backslash\left\{v_{1}, \ldots, v_{4}\right\}$ by a different vertex of $\left\{v_{1}, \ldots, v_{4}\right\}$ that is not contained in $W$. Since 
vertices of $I$ are not incident to the edits of $F^{\prime}$, they are false twins in $G^{\prime} \triangle F^{\prime}$, and hence $W^{\prime}$ created in this manner induces a graph isomorphic to the one induced by $W$. Thus, $W^{\prime}$ is an obstacle in $G^{\prime} \triangle F^{\prime}$. However, the vertices $v_{1}, \ldots, v_{4}$ are not incident to the edits of $F$ and hence $W^{\prime}$ induces the same graph in $G^{\prime} \triangle F^{\prime}$ as in $G^{\prime} \triangle F$. Therefore $W^{\prime}$ would be an obstacle in $G^{\prime} \triangle F$, a contradiction to $G^{\prime} \triangle F$ being trivially perfect.

Since we argued that $F^{\prime} \subseteq F$ is also a solution, by the optimality of $F$ we infer that $F=F^{\prime}$ and $F_{I}=\emptyset$.

We now argue that $G \triangle F$ is trivially perfect, which will imply that $(G, k)$ is a yes-instance. For the sake of contradiction, suppose that there exists an obstacle $W$ in $G \triangle F$; It follows that $W$ shares at least one vertex with $M \backslash I$. From Claim 3.16 it follows that no edit of $F$ is incident to any vertex of $M$, so in $G \triangle F$ we still have that $M$ is a module.

If the obstruction $W$ induces a $P_{4}$, then it is known that $W$ is fully contained in the module $M$, or has at most one vertex in $M[18$, Observation 1]. Since $G[M]=(G \triangle F)[M]$ is trivially perfect, the latter is the case. But since $M$ is a module in $G \triangle F$, then replacing the single vertex of $W \cap A$ with any vertex of $I$ would yield an obstacle in $G^{\prime} \triangle F$, a contradiction.

Consider then the case when $W$ induces a $C_{4}$ in $G \triangle F$. Since $G[M]=(G \triangle F)[M]$ is $C_{4}$-free, we have that $W$ is not entirely contained in $M$. Also, if $W$ had three vertices in $M$, then the remaining vertex would need to be contained in $N_{G}(M)$, and hence would be adjacent in $G \triangle F$ to all the other three vertices of $W$, a contradiction to $(G \triangle F)[W]$ being a $C_{4}$. Therefore, at most two vertices of $W$ can be in $M$.

Suppose exactly two vertices $w_{1}$ and $w_{3}$ of $W$ are in $M$, and $w_{2}$ and $w_{4}$ are outside $M$. As $M$ is a module both in $G$ and in $G \triangle F$, we must have that $w_{2}, w_{4} \in N_{G}(M)$ and hence the 4-cycle induced by $W$ in $G \triangle F$ must be $w_{1}-w_{2}-w_{3}-w_{4}-w_{1}$. Take any two vertices $w_{1}^{\prime}, w_{3}^{\prime} \in I$ and obtain $W^{\prime}$ by replacing $w_{1}$ and $w_{3}$ with them. It follows that $W^{\prime}$ induces a $C_{4}$ in $G^{\prime} \triangle F$, a contradiction.

Finally, consider the case when exactly one vertex of $W$, say $w_{1}$, is in $M$. Again, replacing $w_{1}$ with any vertex of $I$ would yield an induced $C_{4}$ contained in $G^{\prime} \triangle F$, a contradiction. Thus, we conclude that $G \triangle F$ is trivially perfect.

Observe that in order to apply Rule 4, one needs to be given the module $M$. Given $M$, finding any independent set $I \subseteq M$ of size $2 k+4$ can then be done easily as follows: We can find an independent set of maximum cardinality in $M$ in polynomial time, since $G[M]$ is trivially perfect and the INDEPENDENT SET problem is polynomial-time solvable on trivially perfect graphs (it boils down to picking one vertex from every leaf bag of the universal clique decomposition of the considered graph). Then we take any of its subsets of size $2 k+4$ to be $I$. Hence, to apply Rule 4 exhaustively, we need the following statement.

Lemma 3.17. There exists a polynomial-time algorithm that, given an instance $(G, k)$, either finds a module $M \subseteq V(G)$ where Rule 4 can be applied, or correctly concludes that Rule 4 is inapplicable.

Proof. Using Theorem 5 we compute the module decomposition $\left(T,\left(M^{t}\right)_{t \in V(T)}\right)$ of $G$. Then we verify applicability of Rule 4 to each module $M^{t}$ for $t \in V(T)$, by checking whether $G[M]$ is trivially perfect and contains an independent set of size $2 k+5$ (the latter check can be done in polynomial time since $G[M]$ is trivially perfect). Moreover, we perform the same check on all the modules $N_{t}$ formed as follows: take a union node $t \in V(T)$, and construct a module $N_{t}$ by taking the union of labels of those children of $t$ that induce trivially perfect graphs.

We now argue that if Rule 4 is applicable to some module $M$ in $G$, then this algorithm will encounter some (possibly different) module $M^{\prime}$ to which Rule 4 is applicable as well. By the third point of Theorem 5 , either $M=M^{t}$ for some $t \in V(T)$, or $M$ is the union of a collection of labels of children of some union or join node. In the first case the algorithm verifies $M$ explicitly. In the following, let $\alpha(H)$ denote the size of a maximum independent set in a graph $H$.

If now $M$ is a union of labels of some children of a union node $t$, then by heredity $M \subseteq N^{t}$. Moreover, $N^{t}$ induces a trivially perfect graph (since trivially perfect graphs are closed under taking disjoint union) and clearly $\alpha\left(N^{t}\right) \geq \alpha(M)$. Hence, Rule 4 is applicable to $M^{\prime}=N^{t}$, and this will be discovered by the algorithm.

Finally, suppose $M$ is a union of labels of some children $t_{1}, t_{2}, \ldots, t_{p}$ of a join node $t$. Observe that since for every $i \neq j$, every vertex of $M^{t_{i}}$ is adjacent to every vertex of $M^{t_{j}}$, it follows that $\alpha(G[M])=\max _{i=1,2, \ldots, p} \alpha\left(G\left[M^{t_{i}}\right]\right)$. Without loss of generality suppose that the maximum on the 
right hand side is attained for the module $M^{t_{1}}$. Then by heredity $G\left[M^{t_{1}}\right]$ is trivially perfect, and $\alpha\left(G\left[M^{t_{1}}\right]\right)=\alpha(G[M]) \geq 2 k+5$. Therefore Rule 4 is applicable to $M^{\prime}=M^{t_{1}}$, and this will be discovered by the algorithm.

We remark here that for the kernelization algorithm it is not necessary to be sure that Rule 4 is inapplicable at all. Instead, we could perform it on demand. More precisely, during further analysis of the structure of $G-X$ we argue that some modules have to be small, since otherwise Rule 4 would be applicable. This analysis can be performed by a polynomial-time algorithm that would just apply Rule 4 on any encountered module that needs shrinking. However, we feel that the fact that Rule 4 can be indeed applied exhaustively provides a better insight into the algorithm, and streamlines the presentation.

Having introduced and verified Rules 3 and 4, we can now prove that after applying them exhaustively, all the trivially perfect modules in the graph are small.

Lemma 3.18. A (possibly disconnected) trivially perfect graph with maximum true twin class size $t$ and maximum independent set size $\alpha$ has at most $(2 \alpha-1) t$ vertices in total.

Proof. Let $\mathcal{T}$ be the UCD of $G$, a trivially perfect graph with independent set number $\alpha$ and every true twin class of size at most $t$. Since any collection comprising one vertex from each leaf bag of $\mathcal{T}$ forms an independent set, there are at most $\alpha$ leaf bags in $\mathcal{T}$. Thus the number of nodes of $\mathcal{T}$ in total is at most $2 \alpha-1$. Since every bag of the decomposition $T \subseteq V(G)$ is a true twin class, we conclude that there are at most $(2 \alpha-1) t$ vertices in $G$.

Corollary 3.19. Suppose an instance $(G, k)$ is reduced, and moreover Rules 3 and 4 are not applicable to $(G, k)$. Then for every module $M \subseteq V(G)$ such that $G[M]$ is trivially perfect, we have that $|M|=O\left(k^{2}\right)$.

Proof. Suppose $M$ is such a module. Observe that members of every true twin class in $G[M]$ are also true twins in $G$ (since $M$ is a module). Hence twin classes in $G[M]$ have size at most $2 k+4$, as otherwise Rule 3 would be applicable. Moreover, if $G[M]$ contained an independent set of size $2 k+5$, then Rule 4 would be applicable. By Lemma 3.18, we infer that $|M| \leq(4 k+7)(2 k+4)=O\left(k^{2}\right)$.

From now on we assume that in the considered instance $(G, k)$ we have exhaustively applied Rules 1-4, using the algorithms of Lemmas 3.1, 3.14, and 3.17. Hence Corollary 3.19 can be used. Observe that to perform this step, we do not need to construct the small modulator $X$ at all. However, we hope that the reader already sees that Rules $1-4$ will be useful for shrinking too large parts of $G-X$ between the important bags.

\subsection{Kernelizing non-important parts (irrelevant vertex deletion)}

Recall that we have fixed a small TP-modulator $X$ with $|X| \leq 4 k$ such that $G-X$ is a trivially perfect graph with universal clique decomposition $\mathcal{T}$. Moreover, Rules $1-4$ are inapplicable to $(G, k)$. By Lemma 3.8 we have that the number of $X$-neighborhoods is $O\left(k^{4}\right)$. By the marking procedure, we have marked a set $I$ of $O(k)$ bags of $\mathcal{T}$ as important, in such a manner that every connected component of $\mathcal{T}-I$ is adjacent to at most two vertices of $I$, and is in fact of one of the three forms described at the end of Section 3.4.

Thus, the whole vertex set of $G-X$ can be partitioned into four sets:

$V_{I}$ : vertices contained in bags from $I$;

$V_{0}$ : vertices contained in bags of those components of $\mathcal{T}-I$ that are not adjacent to any bag from $I$;

$V_{1}$ : vertices contained in bags of those components of $\mathcal{T}-I$ that are adjacent to exactly one bag from $I$;

$V_{2}$ : vertices contained in bags of those components of $\mathcal{T}-I$ that are adjacent to exactly two bags from $I$.

We are going to establish an upper bound on the cardinality of each of these sets separately. Upper bounds for $V_{I}, V_{0}$, and $V_{1}$ follow already from the introduced reduction rules, but for $V_{2}$ we shall need a new reduction rule. The upper bounds on the cardinalities of $V_{I}$ and $V_{0}$ are quite straightforward.

Lemma 3.20. $\left|V_{I}\right| \leq O\left(k^{6}\right)$. 
Proof. Consider for some $a \in I$ the bag $B_{a}$. Note that $B_{a}$ is a module in $G-X$. By Lemma 3.8 there are only $O\left(k^{4}\right)$ possible $X$-neighborhoods among vertices of $G-X$. Hence, vertices of $B_{a}$ can be partitioned into $O\left(k^{4}\right)$ classes w.r.t. the neighborhoods in $X$. Each such class is a module in $G$ that is also a clique, and hence it is a true twin class. Since the twin reduction rule (Rule 3 ) is not applicable, each true twin class has size at most $2 k+5$, which implies that $\left|B_{a}\right| \leq O\left(k^{5}\right)$. As $|I|=O(k)$, we conclude that $\left|V_{I}\right| \leq O\left(k^{6}\right)$.

We remark that using a more precise analysis of the situation in one bag $B_{a}$ for $a \in I$, one can see that the $X$-neighborhoods of elements of $B_{a}$ are nested, so there is only at most $|X|+1 \leq 4 k+1$ of them. By plugging in this argument in the proof of Lemma 3.20, we obtain a sharper upper bound of $O\left(k^{3}\right)$ instead of $O\left(k^{6}\right)$. However, the upper bounds on $\left|V_{0}\right|$ and $\left|V_{1}\right|$ are $O\left(k^{6}\right)$ and $O\left(k^{7}\right)$, respectively, so establishing a better bound here would have no influence on the overall asymptotic kernel size. Hence, we resorted to a simpler proof of a weaker upper bound.

Lemma 3.21. $\left|V_{0}\right| \leq O\left(k^{6}\right)$.

Proof. Observe that $V_{0}$ is the union of bags of these connected components of $G-X$, whose universal clique decompositions (being components of $\mathcal{T}$ ) do not contain any important bag. By the definition of important bags, each such connected component $C$ is a module in $G$, and clearly its neighborhood is entirely contained in $X$. Recall that by Lemma 3.8 there are only $O\left(k^{4}\right)$ possible different $X$-neighborhoods among vertices of $G-X$. Thus, we can group the connected components of $G\left[V_{0}\right]$ according to their $X$-neighborhoods into $O\left(k^{4}\right)$ groups, and the union of vertex sets in each such group forms a module in $G$. Since Rule 4 is not applicable, by Corollary 3.19 we have that each of these modules has size $O\left(k^{2}\right)$. Thus we infer that $\left|V_{0}\right| \leq O\left(k^{6}\right)$.

To bound the size of $V_{1}$ we need a few more definitions. Suppose that $C$ is a component of $\mathcal{T}-I$ that is adjacent to exactly one important bag $a \in I$. By the construction of $I$, we have that $C$ is a tree rooted in a child of $a$. We shall say that $C$ is attached below $a$. The union of bags of all the components of $\mathcal{T}-I$ attached below $a$ will be called the tassel rooted at a. Thus, $V_{1}$ can be partitioned into $O(k)$ tassels.

Lemma 3.22. For every $a \in I$, the tassel rooted at a has size at most $O\left(k^{6}\right)$.

Proof. Let $C_{1}, C_{2}, \ldots, C_{r}$ be the components of $\mathcal{T}-I$ rooted at the children of $a$, whose union of bags forms the tassel rooted at $a$. Recall that none of the $C_{i}$ s contains any important bag. Therefore, from Lemma 3.10 we infer that for any $C_{i}$ and any $x \in X$, either all the vertices from the bags of $C_{i}$ are adjacent to $x$, or none of them. Thus, the union of bags of each $C_{i}$ forms a module in $G$ : The vertices in this union have the same $X$-neighborhood, and moreover their neighborhoods in $G-X$ are formed by the vertices from the bags on the path from $a$ to the root of $a$ 's connected component in $\mathcal{T}$. Similarly as in the proof of Lemma 3.21, by Lemma 3.8 there are only $O\left(k^{4}\right)$ possible $X$-neighborhoods, so we can partition the components $C_{i}$ into $O\left(k^{4}\right)$ classes with respect to their neighborhoods in $X$. The union of bags in each such class forms a module in $G$; since Rule 4 is not applicable, by Corollary 3.19 we infer that its size is bounded by $O\left(k^{2}\right)$. Thus, the total number of vertices in all the components $C_{i}$ is at most $O\left(k^{6}\right)$.

As $|I|=O(k)$, Lemma 3.22 immediately implies the following.

Lemma 3.23. $\left|V_{1}\right| \leq O\left(k^{7}\right)$.

We are left with bounding the cardinality of $V_{2}$. Let us fix any component $C$ of $\mathcal{T}-I$ which is adjacent in $\mathcal{T}$ to two nodes of $I$. From the construction of $I$, it follows that $C$ has the following form:

- $C$ contains a path $P=a_{1}-a_{2}-\ldots-a_{d}$ such that in $\mathcal{T}$, node $a_{d}$ is a child of an important node $b^{\uparrow}$, and $a_{1}$ has exactly one important child $b^{\downarrow}$.

- For every $i=1,2, \ldots, d, C$ contains also all the subtrees of $\mathcal{T}$ rooted in children of $a_{i}$ that are different from $a_{i-1}\left(\right.$ where $\left.a_{0}=b^{\downarrow}\right)$.

Such a component $C$ will be called a $c o m b$ (see Figure 6). The path $P$ is called the shaft of a comb; the union of the bags of the shaft will be denoted by $Q$. The union of the bags of the subtrees rooted in children of $a_{i}$, apart from $a_{i-1}$, will be called the tooth at $i$, and denoted by $R_{i}$. Note that the subgraph 


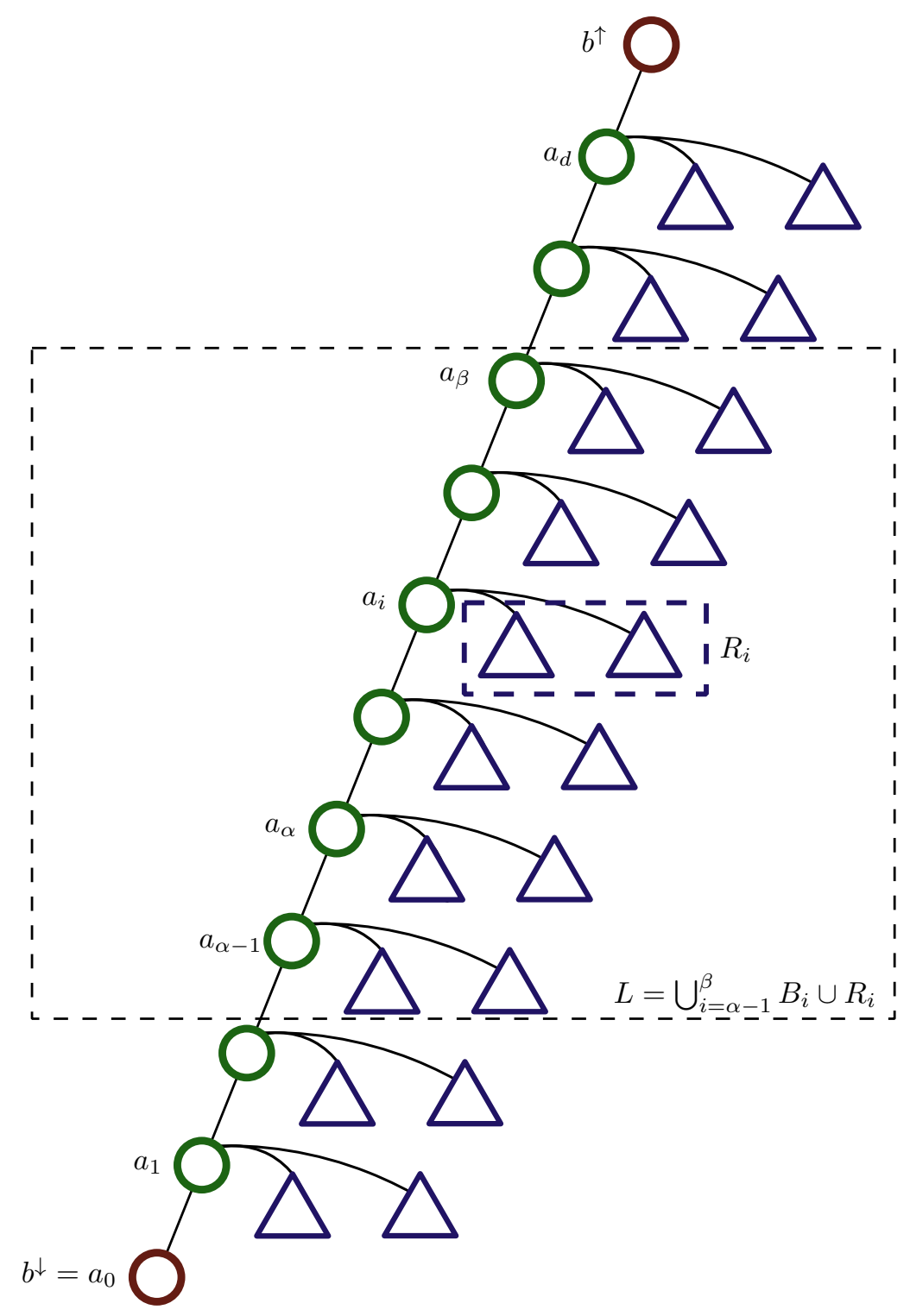

Figure 6: The anatomy of a comb. The top and bottom bags, $b^{\uparrow}$ and $b^{\downarrow}$, are important bags.

induced by a tooth is not necessarily connected; it is, however, always non-empty by the definition of the universal clique decomposition. We also denote $R=\bigcup_{i=1}^{d} R_{i}$. By somehow abusing the notation, we will also denote $B_{i}=B_{a_{i}}$ for $i=1,2, \ldots, d$. The number of teeth $d$ is called the length of a comb.

Since the comb $C$ does not contain any important vertices, from Lemma 3.10 and the construction of $I$ we immediately infer the following observation about the $X$-neighborhoods of vertices of the shaft and the teeth.

Lemma 3.24. There exists two sets $Y, Z$ with $Z \subseteq Y \subseteq X$ such that $N_{X}(u)=Y$ for every $u \in Q$ and $N_{X}(v)=Z$ for every $v \in R$.

In particular, Lemma 3.24 implies that every tooth of a comb is a module. Hence, since Rule 4 is not applicable, we infer that $\left|R_{i}\right|=O\left(k^{2}\right)$ for $i=1,2, \ldots, d$. Also, observe that each $B_{i}$ is a twin class, so by inapplicability of Rule 3 we conclude that $\left|B_{i}\right| \leq 2 k+5$ for each $i=1,2, \ldots, d$.

Since $\mathcal{T}$ is a forest and $|I|=O(k)$, it follows that in $\mathcal{T}-I$ there are $O(k)$ combs. As we already observed, for each comb the sizes of individual teeth and bags on the shaft are bounded polynomially in $k$. Hence, the only thing that remains is to show how to reduce combs that are long. In order to do this, we need one more definition: a tooth $R_{i}$ is called simple if $G\left[R_{i}\right]$ is edgeless, and it is called complicated 
otherwise. We can now state the final reduction rule.

Rule 5. Suppose $C$ is a comb of length at least $(4 k+3)^{2}$, and adopt the introduced notation for the shaft and the teeth of $C$. Define an index $\beta$ as follows:

(i) If at least $4 k+3$ teeth $R_{i}$ are complicated, then we let $\beta=d$.

(ii) Otherwise, there is a sequence of $4 k+3$ consecutive teeth $R_{i}, R_{i+1}, \ldots, R_{i+4 k+2}$ that are simple. Let $\beta$ be the index of the last tooth of this sequence, i.e., $\beta=i+4 k+2$.

Having defined $\beta$, remove the tooth $R_{\beta}$ from the graph and do not modify the budget. That is, proceed with the instance $\left(G-R_{\beta}, k\right)$.

Lemma 3.25. Rule 5 is safe.

Proof. Since $G-R_{\beta}$ is an induced subgraph of $G$, then we trivially have that the existence of a solution for $(G, k)$ implies the existence of a solution for $\left(G-R_{\beta}, k\right)$. Hence, we now prove the converse. Suppose that $F$ is a solution to $\left(G-R_{\beta}, k\right)$, that is, a set of edits in $G-R_{\beta}$ such that $\left(G-R_{\beta}\right) \triangle F$ is trivially perfect and $|F| \leq k$.

We will say that a tooth $R_{i}$ is spoiled if any vertex of $R_{i} \cup B_{i}$ is incident to an edit from $F$, and clean otherwise. The first goal is to find an index $\alpha$ such that

(a) $1<\alpha<\beta$,

(b) the teeth $R_{\alpha-1}$ and $R_{\alpha}$ are clean, and

(c) if any of the teeth $R_{\alpha+1}, R_{\alpha+2}, \ldots, R_{\beta}$ is complicated, then $R_{\alpha}$ is also complicated.

Suppose first that $\beta$ was constructed according to case (i), i.e., there are at least $4 k+3$ complicated teeth in the comb, and hence $\beta=d$. Out of these teeth $R_{i}$, at most one can have index 1 , at most one can have index $d$, at most $2 k$ can be spoiled (since $|F| \leq k$ ) and at most $2 k$ can have the preceding tooth $R_{i-1}$ spoiled. This leaves at least one complicated tooth $R_{i}$ such that $1<i<d$ and both $R_{i}$ and $R_{i-1}$ are clean. Then we can take $\alpha=i$; thus, property (c) of $\alpha$ is satisfied since $R_{\alpha}$ is complicated.

Suppose then that $\beta$ was constructed according to case (ii), i.e., the following teeth are all simple: $R_{\beta-(4 k+2)}, R_{\beta-(4 k+1)}, \ldots, R_{\beta-1}, R_{\beta}$. Similarly as before, out of these $4 k+3$ teeth, one has index $\beta$, one has index $\beta-(4 k+2)$, at most $2 k$ can be spoiled, and at most $2 k$ can have the preceding tooth spoiled. Hence, among them there is a tooth $R_{i}$ such that $\beta-(4 k+2)<i<\beta$ and both $R_{i}$ and $R_{i-1}$ are clean. Again, we take $\alpha=i$; thus, property (c) is satisfied since all the teeth $R_{\beta-(4 k+2)}, R_{\beta-(4 k+1)}, \ldots R_{\beta-1}, R_{\beta}$ are simple.

With $\alpha$ defined, we are ready to complete the proof of Lemma 3.25. To that aim, define $L=$ $\bigcup_{i=\alpha-1}^{\beta} B_{i} \cup R_{i}$. Construct $F^{\prime}$ from $F$ by removing all the edits that are incident to any vertex of $L$; clearly $\left|F^{\prime}\right| \leq|F| \leq k$. We claim that $F^{\prime}$ is a solution to the instance $(G, k)$, that is, that $G \triangle F^{\prime}$ is trivially perfect. For the sake of a contradiction, suppose that $A \subseteq V(G)$ is a vertex set of size 4 such that $G \triangle F^{\prime}[A]$ is a $P_{4}$ or a $C_{4}$. Let $A_{0}=A \cap L$ and $A_{1}=A \backslash A_{0}$.

Claim 3.26. $\left|A_{0}\right|=1$ or $\left|A_{0}\right|=2$.

Proof. Suppose first that $A_{0}=\emptyset$, so $A \subseteq V(G) \backslash L \subseteq V\left(G-R_{\beta}\right)$. Since $F \cap\left(\begin{array}{c}V(G) \backslash L \\ 2\end{array}\right)=F^{\prime} \cap\left(\begin{array}{c}V(G) \backslash L \\ 2\end{array}\right)$ and $R_{\beta} \subseteq L$, we have that the induced subgraph $G \triangle F^{\prime}[A]$ is equal to the induced subgraph $\left(G-R_{\beta}^{2}\right) \triangle F[A]$. However, the graph $\left(G-R_{\beta}\right) \triangle F$ is trivially perfect, so it cannot have an induced $P_{4}$ or $C_{4}$; a contradiction.

Suppose now that $\left|A_{0}\right| \geq 3$. Since $A_{0} \subseteq L$ and no edit of $F^{\prime}$ is incident to any vertex of $L$, we infer that there is no edit of $F^{\prime}$ between vertices of $A$ : only at most one vertex of $A$ does not belong to $A_{0}$. Therefore $G[A]=G \triangle F^{\prime}[A]$ and $G[A]$ is an induced $C_{4}$ or $P_{4}$ in the graph $G$. However, $A_{0} \subseteq L \subseteq V(G) \backslash X$, so $|A \cap X| \leq 1$. Thus, $G[A]$ would be an obstacle in $G$ that has at most one common vertex with TP-modulator $X$, a contradiction with the definition of a TP-modulator (Definition 3.2).

To obtain a contradiction, we shall construct a set $A_{0}^{\prime}$ satisfying the following properties:

(i) $A_{0}^{\prime} \subseteq R_{\alpha-1} \cup B_{\alpha-1} \cup R_{\alpha} \cup B_{\alpha}$; 
(ii) $\left|A_{0}^{\prime}\right|=\left|A_{0}\right|$ and $G\left[A_{0}^{\prime}\right]$ is edgeless if and only if $G\left[A_{0}\right]$ is edgeless;

(iii) $\left|A_{0} \cap Q\right|=\left|A_{0}^{\prime} \cap Q\right|$ and hence $\left|A_{0} \cap R\right|=\left|A_{0}^{\prime} \cap R\right|$.

Let us define $A^{\prime}=A_{1} \cup A_{0}^{\prime}$. For now we postpone the exact construction

Claim 3.27. If $A_{0}^{\prime}$ satisfies properties (i), (ii), and (iii), then $G \triangle F^{\prime}[A]$ is isomorphic to $G \triangle F^{\prime}\left[A^{\prime}\right]$.

Proof. By property (iii) there exists a bijection $\eta$ between $A_{0}$ and $A_{0}^{\prime}$ that preserves belonging to $Q$ or $R$ between the argument and the image. Extend $\eta$ to $A$ by defining $\eta(u)=u$ for $u \in A_{1}$; we claim that $\eta$ is an isomorphism between $G \triangle F^{\prime}[A]$ and $G \triangle F^{\prime}\left[A^{\prime}\right]$. To see this, observe that since $A_{0}, A_{0}^{\prime} \subseteq L$, then we have that no vertex of $A_{0}$ or $A_{0}^{\prime}$ is incident to any edit of $F^{\prime}$. Moreover, in $G$, all the vertices of $L \cap R$ have the same neighborhood in $V(G) \backslash L$, and the same holds also for the vertices of $L \cap Q$. As the neighborhoods of these vertices in $G$ and in $G \triangle F^{\prime}$ are exactly the same, we infer that each vertex $u \in A_{0}$ is adjacent in $G \triangle F^{\prime}$ to the same vertices of $A_{1}$ as the vertex $\eta(u)$ is.

To conclude the proof, we need to prove that $\eta$ restricted to $A_{0}^{\prime}$ is also an isomorphism between $G \triangle F^{\prime}\left[A_{0}\right]$ and $G \triangle F^{\prime}\left[A_{0}^{\prime}\right]$. Again, $A_{0}$ and $A_{0}^{\prime}$ are not incident to any edit of $F^{\prime}$, so $G \triangle F^{\prime}\left[A_{0}\right]=G\left[A_{0}\right]$ and $G \triangle F^{\prime}\left[A_{0}^{\prime}\right]=G\left[A_{0}^{\prime}\right]$. By Claim 3.26 we have that $\left|A_{0}\right|=1$ or $\left|A_{0}\right|=2$, and we conclude by observing that a pair of simple graphs with at most two vertices are isomorphic if and only if both of them are edgeless or both of them contain an edge, and in both cases any bijection between the vertex sets is an isomorphism.

We now argue that the existence of a set $A_{0}^{\prime}$ satisfying properties (i), (ii), and (iii) leads to a contradiction. Recall that the teeth $R_{\alpha-1}$ and $R_{\alpha}$ are clean, which means that no vertex of $R_{\alpha-1} \cup$ $B_{\alpha-1} \cup R_{\alpha} \cup B_{\alpha}$ is incident to any edit from $F$. Moreover, as $\beta>\alpha$, we have that $A^{\prime} \subseteq V\left(G-R_{\beta}\right)$. By the construction of $F^{\prime}$ and $A^{\prime}$ we infer that $G \triangle F^{\prime}\left[A^{\prime}\right]=\left(G-R_{\beta}\right) \triangle F\left[A^{\prime}\right]$. By Claim 3.27 we have that $G \triangle F^{\prime}\left[A^{\prime}\right]$ is a $P_{4}$ or a $C_{4}$, since $G \triangle F^{\prime}[A]$ was. This would, however, mean that $\left(G-R_{\beta}\right) \triangle F$ would contain an induced $P_{4}$ or an induced $C_{4}$, a contradiction to the assumption that $\left(G-R_{\beta}\right) \triangle F$ is trivially perfect.

Therefore, we are left with constructing a set $A_{0}^{\prime}$ satisfying properties (i), (ii), and (iii). We give different constructions depending on the alignment of the vertices of $A_{0}$. In each case we just define $A_{0}^{\prime}$; verifying properties (i), (ii), and (iii) in each case is trivial.

Case 1. $\left|A_{0}\right|=1$.

Case 1a. $A_{0}=\{u\}$ and $u \in Q$. Then $A_{0}^{\prime}=\left\{u^{\prime}\right\}$ for any $u^{\prime} \in B_{\alpha-1}$.

Case 1b. $A_{0}=\{u\}$ and $u \in R$. Then $A_{0}^{\prime}=\left\{u^{\prime}\right\}$ for any $u^{\prime} \in R_{\alpha-1}$.

Case 2. $\left|A_{0}\right|=2$.

Case 2a. $A_{0}=\{u, v\}, u, v \in Q$. As $G[Q]$ is a clique, it follows that $u v \in E(G)$. Then $A_{0}^{\prime}=\left\{u^{\prime}, v^{\prime}\right\}$ for any $u^{\prime} \in B_{\alpha-1}$ and $v^{\prime} \in B_{\alpha}$.

Case 2b. $A_{0}=\{u, v\}, u \in Q, v \in R$, and $u v \notin E(G)$. Then $A_{0}^{\prime}=\left\{u^{\prime}, v^{\prime}\right\}$ for any $u^{\prime} \in B_{\alpha-1}$ and $v^{\prime} \in R_{\alpha}$.

Case 2c. $A_{0}=\{u, v\}, u \in Q, v \in R$, and $u v \in E(G)$. Then $A_{0}^{\prime}=\left\{u^{\prime}, v^{\prime}\right\}$ for any $u^{\prime} \in B_{\alpha}$ and $v^{\prime} \in R_{\alpha-1}$.

Case 2d. $A_{0}=\{u, v\}, u, v \in R$, and $u v \notin E(G)$. Then $A_{0}^{\prime}=\left\{u^{\prime}, v^{\prime}\right\}$ for any $u^{\prime} \in R_{\alpha}$ and $v^{\prime} \in R_{\alpha-1}$.

Case 2e. $A_{0}=\{u, v\}, u, v \in R$, and $u v \in E(G)$. As there are no edges in $G$ between different teeth, we observe that $u, v \in R_{i}$ for some $i$ such that $R_{i} \subseteq L$, i.e., $\alpha-1 \leq i \leq \beta$. In particular, the tooth $R_{i}$ must be complicated. If $i=\alpha-1$ or $i=\alpha$, then we can take $A_{0}^{\prime}=A_{0}$. Otherwise we have that $\alpha<i \leq \beta$ and $R_{i}$ is complicated, so by property (c) of $\beta$ we infer that $R_{\alpha}$ is also complicated. Then we take $A_{0}^{\prime}=\left\{u^{\prime}, v^{\prime}\right\}$ for any $u^{\prime}, v^{\prime} \in R_{\alpha}$ such that $u^{\prime} v^{\prime} \in E(G)$.

This case study is exhaustive due to Claim 3.26.

We can finally gather all the pieces and prove our main theorem. 
Theorem 6. The problem TRIVIALly PERfECT EDITING admits a proper kernel with O $\left(k^{7}\right)$ vertices.

Proof. The algorithm first applies Reduction Rules $1-4$ exhaustively. As each application of a reduction rule either decreases $n$ and does not change $k$, or decreases $k$ while not changing $n$, the number of applications of these rules will be bounded by $O(n+k)$ until $k$ becomes negative and we can conclude that we are working with a no-instance. By Lemmas 3.1, 3.14, 3.15, and 3.17, these rules are safe, applicability of each rule can be recognized in polynomial time, and applying the rules also takes polynomial time.

After Rules 1-4 have been applied exhaustively, we construct a small TP-modulator $X$ using the algorithm of Lemma 3.3. In case the construction fails, we conclude that we are working with a no-instance. Otherwise, in polynomial time we construct the universal clique decomposition $\mathcal{T}$ of $G-X$, and then we mark the set $I$ of important bags. Both locating the important bags and performing the lowest common ancestor closure can be done in polynomial time. After this, we examine all the combs of $\mathcal{T}-I$. In case there is a comb of length greater than $(4 k+3)^{2}$, we apply Rule 5 on it and restart the whole algorithm. Observe that each application of this rule reduces the vertex count by one while keeping $k$, so the total number of times the algorithm is restarted is bounded by the vertex count of the original instance.

We are left with analyzing the situation when Reduction Rule 5 is not applicable, i.e., all the combs have length less than $(4 k+3)^{2}$. As we have argued, the inapplicability of Rules 3 and 4 ensures that bags of shafts of combs have sizes $O(k)$ and teeth of combs have sizes $O\left(k^{2}\right)$. Hence, every comb has $O\left(k^{4}\right)$ vertices. Since the number of combs is $O(k)$, we infer that $\left|V_{2}\right| \leq O\left(k^{5}\right)$. Together with the upper bounds on the sizes of $V_{I}, V_{0}$, and $V_{1}$ given by Lemmas $3.20,3.21$, and 3.23 , we conclude that

$$
|V(G)|=|X|+\left|V_{I}\right|+\left|V_{0}\right|+\left|V_{1}\right|+\left|V_{2}\right| \leq 4 k+O\left(k^{6}\right)+O\left(k^{6}\right)+O\left(k^{7}\right)+O\left(k^{5}\right)=O\left(k^{7}\right) .
$$

Hence, we can output the current instance as the obtained kernel.

\section{Kernels for Trivially Perfect Completion/Deletion}

We now present how the technique applied to Trivially Perfect Editing also yields polynomial kernels for Trivially Perfect Completion and Trivially Perfect Deletion after minor modifications. That is, we prove Theorems 2 and 3 .

We show that all the rules given above, with only two minor modifications are correct for both problems. Clearly, the running times of the algorithms recognizing applicability of the rule do not depend on the problem we are solving, so we only need to argue for their safeness.

In the first two rules, Rules 1 and 2, we add and delete an edge, respectively, and the argument is that any editing set of size at most $k$ must necessarily include this edit. However, in the completion and deletion version, we are not allowed both operations. Hence, for the first rule, in the deletion variant we can immediately infer that we are working with a no-instance, and respectively for the second rule in the completion variant.

Thus, the two following rules replace Rule 1 for deletion and Rule 2 for completion, and their safeness is guaranteed by a trivial modification of the proof of Lemma 3.1:

Rule 1D. For an instance $(G, k)$ with $u v \notin E(G)$, if there is a matching of size at least $k+1$ in $\overline{G[N(u) \cap N(v)]}$, then return a trivial no-instance as the computed kernel.

Rule 2C. For an instance $(G, k)$ with $u v \in E(G)$ and $N_{1}=N(u) \backslash N[v]$ and $N_{2}=N(v) \backslash N[u]$, if there is a matching in $\bar{G}$ between $N_{1}$ and $N_{2}$ of size at least $k+1$, then return a trivial no-instance as the computed kernel.

Observe that Rules $1 \mathrm{D}$ and $2 \mathrm{C}$ are applicable in exactly the same instances as their unmodified variants. Hence, exhaustive application of the basic rules with any of these modifications results in exactly the same notion of a reduced instance as the one introduced in Section 3.1. We now argue that Rules 3 and 4 are safe for both the deletion and the completion variant, without any modifications.

Lemma 4.1. Rules 3 and 4 are safe both for Trivially PERfect Deletion and for Trivially Perfect Completion.

Proof. The proof of the safeness of Rule 3 (Lemma 3.14) in fact argues that every editing set $F$ for $(G-v, k)$ with $|F| \leq k$ is also an editing set for $(G, k)$. This holds also for editing sets that consist only 
of edge additions/deletions, so the reasoning remains the same for TRIVIALly PERfECT Deletion and Trivially Perfect Completion.

The proof of the safeness of Rule 4 (Lemma 3.15) first argues that any minimum-size editing set $F$ for the reduced instance $\left(G^{\prime}, k\right)$ is not incident to any vertex of $I$. This is done by showing that otherwise $F$ would not be an inclusion-wise minimal editing set (proof of Claim 3.16), and the argumentation can be in the same manner applied to minimum-size completion/deletion sets. Then it is argued that $F$ is in fact an editing set for the original instance $(G, k)$, and the argumentation is oblivious to whether $F$ is allowed to contain edge additions or deletions.

We now proceed to the analysis of Rule 5 in the completion and deletion variants. First, let us consider the construction of the modulator. In the completion/deletion variants we can construct the modulator in exactly the same manner as for editing. Indeed, the main argument for the bound $|X| \leq 4 k$ states that if the construction was performed for more than $k$ rounds, then we are dealing with a no-instance, since then any editing set for $G$ has size at least $k+1$. Completion and deletion sets are editing sets in particular, so the same argument holds also for Trivially Perfect Deletion and Trivially Perfect Completion.

Results of Sections 3.3 and 3.4, i.e., the analysis of the $X$-neighborhoods and marking of the important bags, work in exactly the same manner, since they are based on the same notions of a reduced instance and of a TP-modulator. Thus, Lemma 3.8 holds as well, and we have marked the same set $I$ of $O(k)$ important bags, with the same properties. Rules 3 and 4 are not modified, so the bounds on $\left|V_{I}\right|,\left|V_{0}\right|$ and $\left|V_{1}\right|$ from Lemmas 3.20, 3.21, and 3.23 also hold.

We are left with analyzing Rule 5, and we claim that this rule is also safe for Trivially PERfECT Deletion and Trivially Perfect Completion without any modifications. Indeed, in the proof of the safeness of the rule (Lemma 3.25), we have argued that for every editing set $F(|F| \leq k)$ for the new instance $\left(G^{\prime}, k\right)$, there exists some $F^{\prime} \subseteq F$ which is a solution to the original instance $(G, k)$. In case $F$ consists of edge deletions or edge additions only, so does $F^{\prime}$. Hence, $\left(G^{\prime}, k\right)$ being a yes-instance of Trivially Perfect Deletion, resp. Trivially Perfect Completion, implies that $(G, k)$ is also a yes-instance of the same problem. Thus Rule 5 is safe without any modifications, and the kernel size analysis contained in the proof of Theorem 6 (end of Section 3.6) can be performed in exactly the same manner. This concludes the proof of Theorems 2 and 3.

\section{$5 \quad$ Hardness results}

In this section we show that TRIVIAlly PERFECT EDITING is NP-hard, and furthermore not solvable in subexponential parameterized time unless the Exponential Time Hypothesis fails. Recall that the NP-hardness of the problem was already established by Nastos and Gao [27]. Their reduction (see the proof of Theorem 3.3 in [27]) starts with an instance of EXACT 3-COVER with universe of size $n$ and set family of size $m$, and constructs an instance $(G, k)$ of Trivially Perfect Editing with $k=\Theta\left(m n^{2}\right)$. Thus, the parameter blow-up is at least cubic, and the reduction cannot be used to establish the non-existence of a subexponential parameterized algorithm under ETH.

Here, we give a direct, linear reduction from 3SAT to Trivially Perfect Editing. Furthermore, the resulting graph in our reduction has maximum degree equal to 4 . Thus, we in fact prove that even on input graphs of maximum degree 4, Trivially PERfECT EDITING remains NP-hard and does not admit a subexponential parameterized algorithm, unless ETH fails. Formally, the following theorem will be proved, where for an input formula $\varphi$ of $3 \mathrm{SAT}$, by $\mathcal{V}(\varphi)$ and $\mathcal{C}(\varphi)$ we denote the variable and clause sets of $\varphi$, respectively:

Theorem 7. There exists a polynomial-time reduction that, given an instance $\varphi$ of 3SAT, returns an equivalent instance $\left(G_{\varphi}, k_{\varphi}\right)$ of Trivially Perfect Editing, where $\left|V\left(G_{\varphi}\right)\right|=13|\mathcal{C}(\varphi)|,\left|E\left(G_{\varphi}\right)\right|=$ $18|\mathcal{C}(\varphi)|, k_{\varphi}=5|\mathcal{C}(\varphi)|$, and $\Delta\left(G_{\varphi}\right)=4$. Consequently, even on instances with maximum degree 4 , TRIVIALly Perfect Editing remains NP-hard and cannot be solved in time $2^{o(k)} n^{O(1)}$ or $2^{o(n+m)}$, unless ETH fails.

Theorem 7 clearly refines Theorem 4, and its conclusion follows from the reduction by an application of Proposition 2.2. Hence, we are left with constructing the reduction, to which the rest of this section is devoted. Our approach is similar to the technique used by Komusiewicz and Uhlmann to show the 


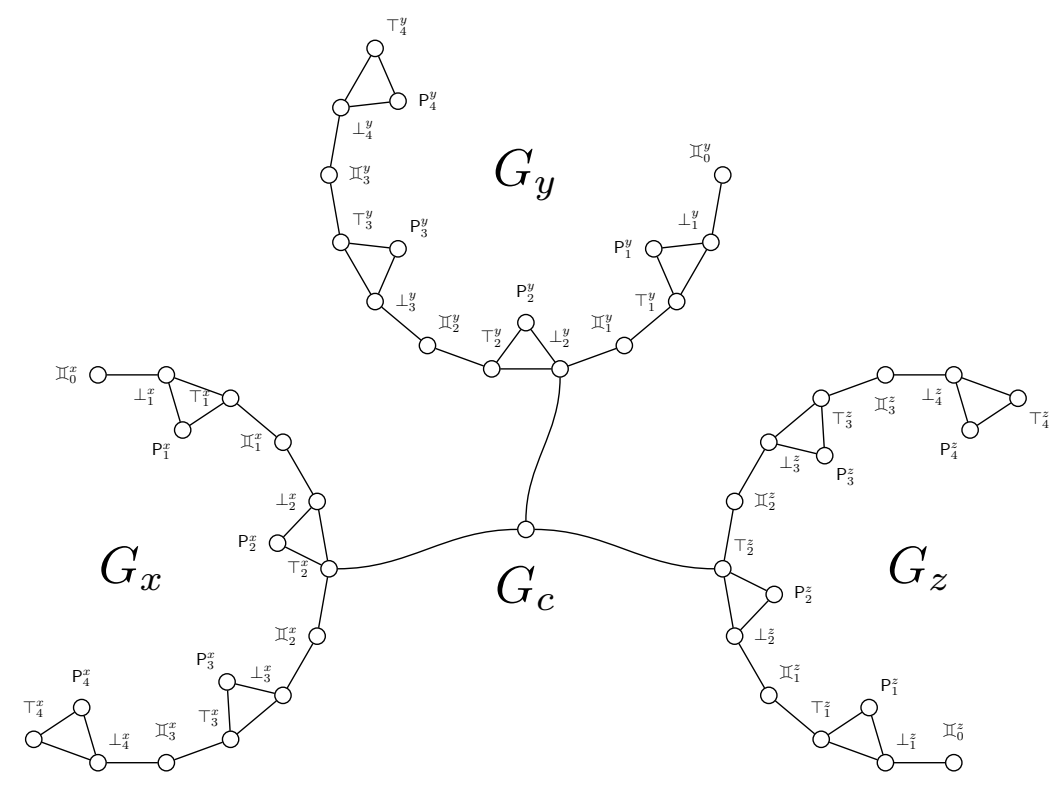

Figure 7: Gadget $c=x \vee \neg y \vee z$. The clause $c$ is now the second clause all variables $x, y$, and $z$ appear in, and $x$ and $z$ appears positively whereas $y$ appears negatively.

hardness of a similar problem, Cluster Editing [22]; However, the gadgets are heavily modified to work for the Trivially Perfect Editing problem.

Let $\varphi$ be the input instance of 3SAT. By standard modifications of the formula we may assume that every clause contains exactly three literals, all containing different variables, and that every variable appears in at least two clauses. For a variable $x \in \mathcal{V}(\varphi)$, let $p_{x}>1$ be the number of occurrences of $x$ in the clauses of $\varphi$; Moreover, we order these occurrences arbitrarily. Observe that $\sum_{x \in \mathcal{V}(\varphi)} p_{x}=3|\mathcal{C}(\varphi)|$. Now, for every $x \in \mathcal{V}(\varphi)$ we create a variable gadget, and for every $c \in \mathcal{C}(\varphi)$ we create a clause gadget.

Variable gadgets. For $x \in \mathcal{V}(\varphi)$, construct a graph $G_{x}$ isomorphic to $C_{3 p_{x}}$, a cycle on $3 p_{x}$ vertices. The vertices of $G_{x}$ are labeled $\perp_{i}^{x}, \boldsymbol{\top}_{i}^{x}$, 邛 $i_{i}^{x}$ for $i \in\left[0, p_{x}-1\right]$, in the order of their appearance on the cycle. We then add a vertex $\mathrm{P}_{i}^{x}$ adjacent to $\top_{i}^{x}$ and $\perp_{i}^{x}$, for each $i \in\left[0, p_{x}-1\right]$, see Figure 7 . Formally, the vertices $\mathrm{P}_{i}^{x}$ do not belong to $G_{x}$, but they will be used to wire variable gadgets with clause gadgets. This concludes the construction of the variable gadget, and it should be clear that the number of created vertices and edges is bounded linearly in $p_{x}$; More precisely, we created $4 p_{x}$ vertices and $5 p_{x}$ edges.

For the sake of later argumentation, we now define the deletion set $F_{x}^{\alpha}$ for $G_{x}$. If, in an assignment of variables $\alpha: \mathcal{V}(\varphi) \rightarrow\{\top, \perp\}$, we have $\alpha(x)=\top$, then we let $F_{x}^{\alpha}$ be the set consisting of every edge of the form $\mathbb{I}_{i}^{x} \perp_{i+1}^{x} \bmod p_{x}$ for $i \in\left[0, p_{x}-1\right]$. If, on the other hand, $\alpha(x)=\perp$, we define the deletion set $F_{x}^{\alpha}$ to be the set comprising the edges $\top_{i}^{x} \widetilde{\amalg}_{i}^{x}$ for $i \in\left[0, p_{x}-1\right]$, see Figure 8 . We will later show that these are the only relevant editing sets of size at most $p_{x}$ for $G_{x}$.

Clause gadget. The clause gadgets are very simple. A clause gadget consists simply of one vertex, i.e., for a clause $c \in \mathcal{C}(\varphi)$ construct the vertex $v_{c}$. This vertex will be connected to $G_{x}, G_{y}$ and $G_{z}$, for $x, y$, and $z$ being the variables appearing in $c$, in appropriate places, depending on whether the variable occurs positively or negatively in $c$. More precisely, if $c$ is the $i$ th clause $x$ appears in, then we make $v_{c}$ adjacent to $\top_{i}^{x}$ provided that $x$ appears positively in $c$, and to $\perp_{i}^{x}$ provided that $x$ appears negatively in $c$. This concludes the construction of a clause gadget. As every clause gadget contains one vertex and three edges, the construction of all the clause gadgets creates $|\mathcal{C}(\varphi)|$ vertices and $3|\mathcal{C}(\varphi)|$ edges.

The deletion set for a clause gadget will be as follows. Let $\alpha: \mathcal{V}(\varphi) \rightarrow\{\top, \perp\}$, be an assignment of the variables that satisfies all the clauses. Suppose $c=\ell_{x} \vee \ell_{y} \vee \ell_{z}$, where the literals $\ell_{x}, \ell_{y}$, and $\ell_{z}$ contain variables $x, y$, and $z$, respectively. Pick any literal satisfying $c$, say $\ell_{x}$, and delete the two other edges in the connection, i.e., the two edges connecting $v_{c}$ with vertices of $G_{y}$ and $G_{z}$. Thus $v_{c}$ remains a vertex of degree 1, adjacent to a vertex of $G_{x}$. 


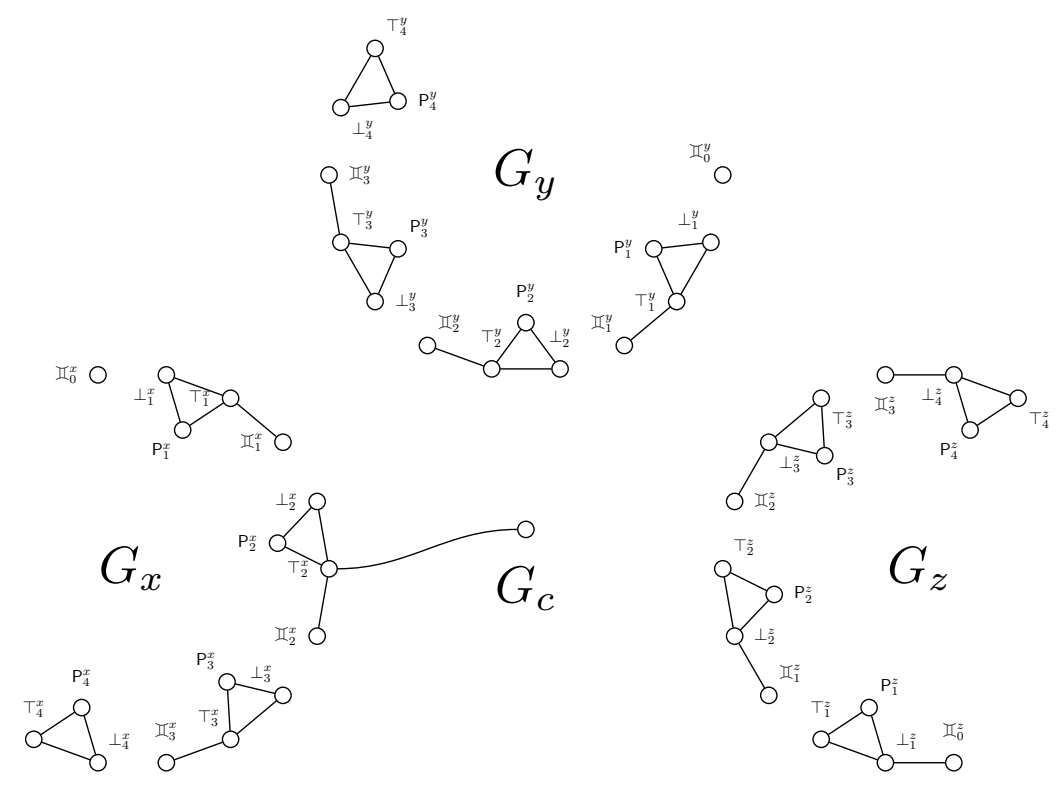

Figure 8: Edited gadget of $c=x \vee \neg y \vee z$ where $\alpha(x)=\top, \alpha(y)=\top$ and $\alpha(z)=\perp$ and $x$ has been chosen (no choice) to satisfy $c$. Notice the formation of paws, except the one incident to $c$ which induces a cricket.

Let $G_{\varphi}$ be the constructed graph. We set the budget for edits to

$$
k_{\varphi}=\sum_{x \in \mathcal{V}(\varphi)} p_{x}+2|\mathcal{C}(\varphi)|=5|\mathcal{C}(\varphi)| .
$$

Observe also that

$$
\begin{aligned}
& \left|V\left(G_{\varphi}\right)\right|=\sum_{x \in \mathcal{V}(\varphi)} 4 p_{x}+|\mathcal{C}(\varphi)|=13|\mathcal{C}(\varphi)|, \\
& \left|E\left(G_{\varphi}\right)\right|=\sum_{x \in \mathcal{V}(\varphi)} 5 p_{x}+3|\mathcal{C}(\varphi)|=18|\mathcal{C}(\varphi)|,
\end{aligned}
$$

and that $\Delta\left(G_{\varphi}\right)=4$. Thus, all the technical properties stated in Theorem 7 are satisfied, and we are left with proving that $\left(G_{\varphi}, k_{\varphi}\right)$ is a yes-instance of Trivially Perfect Editing if and only if $\varphi$ is satisfiable.

Before we state the main lemma, we give two auxiliary observations that settle the tightness of the budget:

Claim 5.1. Suppose that a graph $H$ is a cycle on $3 p$ vertices for some $p>1$, and suppose $F$ is an editing set for $H$. Then $|F| \geq p$. Moreover, if $|F|=p$ then $F$ consists of deletions of every third edge of the cycle.

Claim 5.2. Suppose a graph $H$ is a subdivided claw, i.e., the star $K_{1,3}$ with every leg subdivided once (see Figure 9a). Furthermore, suppose that $F$ is an editing set for $H$. Then $|F| \geq 2$. Moreover, if $|F|=2$ then $F$ consists of deletions of two edges incident to the center of the subdivided claw (see Figure 9b).

We will prove the two claims in order now. The astute reader should already see that this implies the tightness of the budget: every editing set needs to include exactly $p_{x}$ edges of every variable gadget $G_{x}$ (by Claim 5.1), and exactly two edges incident to every vertex $v_{c}$ (by Claim 5.2). The additional vertices $\mathrm{P}_{i}^{x}$ will form the degree- 1 vertices of subdivided claws created by clause gadgets, and all the subgraphs in question pairwise share at most single vertices, which means that any edit can influence at most one of them. This statement is made formal in the proof of Lemma 5.3.

Proof of Claim 5.1. Let $v_{0}, v_{1}, \ldots, v_{3 p-1}$ be the vertices of $H$, in their order of appearance on the cycle. For $i=0,1, \ldots, p-1$, let $A_{i}=\left\{v_{3 i}, v_{3 i+1}, v_{3 i+2}, v_{3 i+3}\right\}$; Here and in the sequel, the indices behave cyclically in a natural manner. Observe that each $A_{i}$ induces a $P_{4}$ in $H$, hence $F \cap\left(\begin{array}{c}A_{i} \\ 2\end{array}\right) \neq \emptyset$. However, the sets $\left(\begin{array}{c}A_{i} \\ 2\end{array}\right)$ are pairwise disjoint for $i=0,1, \ldots, p-1$, from which it follows that $|F| \geq p$. 
Suppose now that $|F|=p$. Hence $\left|F \cap\left(\begin{array}{c}A_{i} \\ 2\end{array}\right)\right|=1$ for each $i \in[0, p-1]$, and there are no edits outside the sets $\left(\begin{array}{c}A_{i} \\ 2\end{array}\right)$. There are five possible ways for an $A_{i}$ of how $F \cap\left(\begin{array}{c}A_{i} \\ 2\end{array}\right)$ can look like: It is either a deletion of the edge $v_{3 i} v_{3 i+1}, v_{3 i+1} v_{3 i+2}$, or $v_{3 i+2} v_{3 i+3}$ (henceforth referred to as types $D^{-}, D^{0}$, and $D^{+}$, respectively), or an addition of the edge $v_{3 i} v_{3 i+2}$ or $v_{3 i+1} v_{3 i+3}$ (henceforth called types $C^{-}$and $C^{+}$, respectively) - the sixth possibility, which has been left out, creates an induced $C_{4}$. Observe now that if some $A_{i}$ has type $D^{-}$, then $A_{i+1}$ also has type $D^{-}$, or otherwise a $P_{4} v_{3 i+1}-v_{3 i+2}-v_{3 i+3}-v_{3 i+4}$ would remain in the graph. Similarly, if $A_{i}$ has type $D^{+}$then $A_{i-1}$ also has type $D^{+}$. Hence, if type $D^{+}$or $D^{-}$ appears for any $A_{i}$, then all the $A_{i}$ s have the same type. Observe now that if some $A_{i}$ had type $C^{-}$ and $C^{+}$, then $A_{i-1}$ would have to have type $D^{+}$and $A_{i+1}$ would have to have type $D^{-}$or otherwise an unresolved $P_{4}$ would appear; This is a contradiction with the previous observations, since types $D^{-}$ and $D^{+}$cannot appear simultaneously. Hence, we are left with only three possibilities: all the $A_{i}$ s have type $D^{-}$, or all have type $D^{0}$, or all have type $D^{+}$.

Proof of Claim 5.2. Denote the vertices of $H$ as in Figure 9a. Consider the following three $P_{4}$ s in $H$ :

- $a_{2}-a_{1}-v-c_{1}$,

- $b_{2}-b_{1}-v-a_{1}$, and

- $c_{2}-c_{1}-v-b_{1}$.

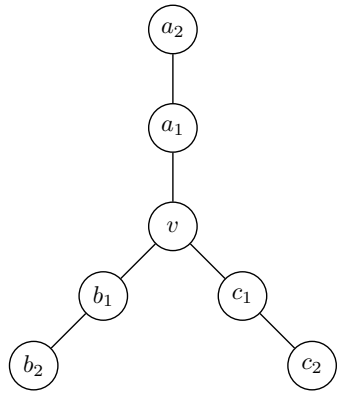

(a) A subdivided claw.

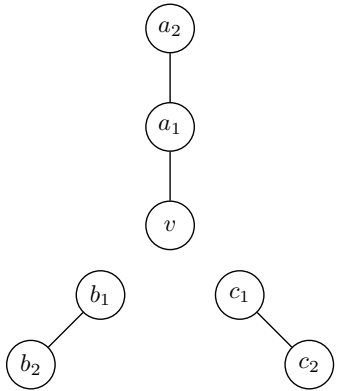

(b) An optimally edited subdivided claw.

Observe that any edge addition in $H$ can destroy at most one of these $P_{4}$ s, and a deletion of any of edges $a_{1} a_{2}, b_{1} b_{2}$, or $c_{1} c_{2}$ also can destroy at most one of these $P_{4}$ s. Moreover, a deletion of any of the edges incident to the center $v$ destroys only two of them. We infer that $|F| \geq 2$ since no single edit can destroy all three considered $P_{4} \mathrm{~s}$, and moreover if $|F|=2$, then $F$ contains at least one deletion of an edge incident to $v$, say $v a_{1}$. After deleting this edge we are left with a $P_{5} b_{2}-b_{1}-v-c_{1}-c_{2}$, and it can be readily checked that the only way to edit it to a trivially perfect graph using only one edit is to delete $v b_{1}$ or $v c_{1}$. Thus, any editing set $F$ with $|F|=2$ in fact consists of deletions of two edges incident to $v$.

Lemma 5.3. The input 3SAT instance $\varphi$ is satisfiable if and only if $\left(G_{\varphi}, k_{\varphi}\right)$ is a yes-instance of Trivially Perfect Editing.

Proof. Suppose $\varphi$ is satisfiable and let $\alpha: \mathcal{V}(\varphi) \rightarrow\{\top, \perp\}$ be a satisfying assignment. Define editing set $F^{\alpha}=\bigcup_{x \in \mathcal{V}(\varphi)} F_{x}^{\alpha} \cup \bigcup_{c \in \mathcal{C}(\varphi)} F_{c}^{\alpha}$; Note that $F$ consists of deletions only. Then we have that $\left|F^{\alpha}\right|=k_{\varphi}$ and it can be easily seen that $G \triangle F$ is a disjoint union of components of constant size, each being a paw or a cricket (see Figure 9). Both these graphs are trivially perfect, so a disjoint union of any number of their copies is also a trivially perfect graph. Thus $F^{\alpha}$ is a solution to the instance $\left(G_{\varphi}, k_{\varphi}\right)$.

For the other direction, let $F \subseteq\left(\begin{array}{c}V\left(G_{\varphi}\right) \\ 2\end{array}\right)$ be an editing set such that $G_{\varphi} \triangle F$ is trivially perfect, and $|F| \leq k_{\varphi}$. For every $x \in \mathcal{V}(\varphi)$ consider the subgraph $G_{x}$. For every $c \in \mathcal{C}(\varphi)$ consider the subgraph $G_{c}$ induced in $G$ by

- vertex $v_{c}$;

- the three neighbors of $v_{c}$, say $\square_{i_{x}}^{x}, \square_{i_{y}}^{y}$, and $\square_{i_{z}}^{z}$, where $x, y, z$ are variables appearing in $c$ and each symbol $\square$ is replaced by $\perp$ or $\top$ depending whether the variable's occurrence is positive or negative; and 


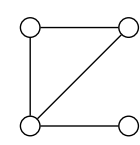

(c) Paw

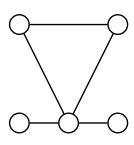

(d) Cricket

Figure 9: Shapes of components of $G$ after editing deletion sets $F_{x}^{\alpha}$ and $F_{c}^{\alpha}$ for $\alpha$ being a satisfying assignment. Both of them are trivially perfect, so a disjoint union of any number of their copies is also trivially perfect.

- vertices $\mathrm{P}_{i_{x}}^{x}, \mathrm{P}_{i_{y}}^{y}$, and $\mathrm{P}_{i_{z}}^{z}$.

Observe that each $G_{x}$ is isomorphic to a cycle on $3 p_{x}$ vertices and each $G_{c}$ is isomorphic to a subdivided claw. Moreover, all these subgraphs pairwise share at most one vertex, which means that sets $\left(\begin{array}{c}V\left(G_{x}\right) \\ 2\end{array}\right)$ for $x \in \mathcal{V}(\varphi)$ and $\left(\begin{array}{c}V\left(G_{c}\right) \\ 2\end{array}\right)$ for $c \in \mathcal{C}(\varphi)$ are pairwise disjoint. By Claim 5.1 we infer that $\left|F \cap\left(\begin{array}{c}V\left(G_{x}\right) \\ 2\end{array}\right)\right| \geq p_{x}$ for each $x \in \mathcal{V}(\varphi)$, and by Claim 5.2 we infer that $\left|F \cap\left(\begin{array}{c}V\left(G_{c}\right) \\ 2\end{array}\right)\right| \geq 2$ for each $c \in \mathcal{C}(\varphi)$. Thus

$$
|F| \geq \sum_{x \in \mathcal{V}(\varphi)} p_{x}+2|\mathcal{C}(\varphi)|=k_{\varphi}
$$

Hence, in fact $|F|=k_{\varphi}$ and all the used inequalities are in fact equalities: $\left|F \cap\left({ }^{V\left(G_{x}\right)}\right)\right|=p_{x}$ for each $x \in \mathcal{V}(\varphi)$ and $\left|F \cap\left(\begin{array}{c}V\left(G_{c}\right) \\ 2\end{array}\right)\right|=2$ for each $c \in \mathcal{C}(\varphi)$. Using Claims 5.1 and 5.2 again, we infer that $F$ has the following form: it consists of deletions only, from every cycle $G_{x}$ it deletes every third edge, and for every vertex $v_{c}$ it deletes two out of three edges incident to it. In particular, no edit is incident to any of the vertices $\mathrm{P}_{i}^{x}$ for $x \in \mathcal{V}(\varphi)$ and $i \in\left[0, p_{x}-1\right]$.

Consider now the cycle $G_{x}$; We already know that the solution deletes either all the edges $\perp_{i}^{x} \top_{i}^{x}$ for $i \in\left[0, p_{x}-1\right]$, or all the edges $\top_{i}^{x} \mathbb{Z}_{i}^{x}$ for $i \in\left[0, p_{x}-1\right]$, or all the edges $\mathbb{I}_{i}^{x} \perp_{i+1}^{x} \bmod p_{x}$ for $i \in\left[0, p_{x}-1\right]$. Observe that the first case cannot happen, since then we would have an induced $P_{4} \perp_{i}^{x}-\mathrm{P}_{i}^{x}-\mathrm{T}_{i}^{x}-\mathbb{I}_{i}^{x}$ remaining in the graph — no other edit can destroy it. Hence, one of the latter two cases happen. Construct an assignment $\alpha: \mathcal{V}(\varphi) \rightarrow\{\top, \perp\}$ by, for each $x \in \mathcal{V}(\varphi)$, putting $\alpha(x)=\perp$ if all the edges $\top_{i}^{x} \mathbb{\Pi}_{i}^{x}$ are included in $F$, and $\alpha(x)=\top$ if all the edges $\mathbb{I}_{i}^{x} \perp_{i+1}^{x} \bmod p_{x}$ are included in $F$. We now claim that $\alpha$ satisfies $\varphi$.

For the sake of contradiction, suppose that a clause $c=\ell_{x} \vee \ell_{y} \vee \ell_{z}$ is not satisfied by $\alpha$. Let $e$ be the edge incident to $v_{c}$ which has not been removed and suppose without loss of generality that this edge connects $v_{c}$ with $G_{x}$. Suppose further that $\ell_{x}=x$, i.e., $x$ appears positively in $c$, so $e=v_{c} \top_{i}^{x}$ for some $i \in\left[0, p_{x}-1\right]$. Since $x$ does not satisfy $c, \alpha(x)=\perp$ and both edges $\Pi_{i-1 \bmod p_{x}}^{x} \perp_{i}^{x}$ and $\perp_{i}^{x} \top_{i}^{x}$ are not deleted in $F$ - the deleted edge is $\top_{i}^{x} \overleftarrow{I}_{i}^{x}$. But then we have the following induced $P_{4}$ : $v_{c}-\top_{i}^{x}-\perp_{i}^{x}-\mathbb{I}_{i-1}^{x} \bmod p_{x}$, which contradicts the assumption that $G_{\varphi} \triangle F$ is trivially perfect. The case when $\ell_{x}=\neg x$, i.e., $x$ appears negatively in $c$, is symmetric.

Hence $\alpha$ is indeed a satisfying assignment for $\varphi$ and we are done.

Lemma 5.3 guarantees that the reduction is correct, and hence Theorem 7 follows by a straightforward application of Proposition 2.2. We can also observe that this reduction works immediately for TRIVIALLY Perfect Deletion as well since every optimal edit set consisted purely of deletions (see Claims 5.1 and 5.2), however this result is known [9].

\section{Conclusion}

In this paper we gave the first polynomial kernels for Trivially Perfect Editing and Trivially Perfect Deletion, which answers an open problem by Nastos and Gao [27], and Liu, Wang, and Guo [24]. We also proved that assuming ETH, Trivially PERfect Editing does not have a subexponential parameterized algorithm. Together with the earlier results $[9,19]$, we thus obtain a complete picture of the existence of polynomial kernels and subexponential parameterized algorithms for edge modification problems related to trivially perfect graphs; see Figure 10 for an overview. In particular, the fact that all three problems Trivially Perfect Editing, Trivially Perfect Completion, and Trivially Perfect Deletion admit polynomial kernels, stands in an interesting contrast with the results of Cai 
and Cai [6], who showed that this is not the case for any of $C_{4}$-Free Editing, $C_{4}$-Free Completion and $C_{4}$-Free Deletion.

The main contribution of the paper is the proof that TRIVIALly PERFECT EDITING admits a polynomial kernel with $O\left(k^{7}\right)$ vertices. We apply the existing technique of constructing a vertex modulator, but with a new twist: The fact that we are solving an edge modification problem enables us also to argue about the adjacency structure between the modulator and the rest of the graph, which is helpful in understanding the structure of the instance. We believe that this new insight can be applied to other edge modification problems as well.

Finally, we showed that Trivially Perfect Editing, in addition to being NP-complete, is not solvable in subexponential parameterized time unless the Exponential Time Hypothesis fails. The same result was known for TRIVIALly PERFECT DELETION, but contrasts the previous result that the completion variant does admit a subexponential parameterized algorithm [9].

\begin{tabular}{lll} 
Problem & Polynomial kernel & Subexp. par. algorithm \\
\hline & & \\
Trivially Perfect Completion & Yes [19] & Yes [9] \\
Trivially Perfect Deletion & Yes & No [9] \\
Trivially Perfect Editing & Yes & No
\end{tabular}

Figure 10: Graph modification problems related to trivially perfect graphs

Let us conclude by stating some open questions. In this paper, we focused purely on constructing a polynomial kernel for TRIVIALly PERFECT EDITING and related problems, and in multiple places we traded possible savings in the overall kernel size for simpler arguments in the analysis. We expect that a tighter analysis of our approach might yield kernels with $O\left(k^{6}\right)$ or even $O\left(k^{5}\right)$ vertices, but we think that the really challenging question is to match the size of the cubic kernel for Trivially PERFECT Completion of Guo [19].

Generally, we find the vertex modulator technique very well-suited for tackling kernelization of edge modification problems, since it is at the same time versatile, and exposes well the structure of a large graph that is close in the edit distance to some graph class. We have high hopes that this generic approach will find applications in other edge modification problems as well, both in improving the sizes of existing kernels and in finding new positive results about the existence of polynomial kernels. For concrete questions where the technique might be applicable, we propose the following:

- Is it possible to improve the $O\left(k^{3}\right)$ vertex kernels for Cograph Editing and Cograph Completion of Guillemot et al. [18]?

- Is it possible to improve the $O\left(k^{4}\right)$ vertex kernel for the Split Deletion problem of Guo [19]?

- Do the Claw-Free Edge Deletion or Line Graph Edge Deletion problems admit polynomial kernels? Here, the task is to remove at most $k$ edges to obtain a graph that is claw-free, i.e., does not contain $K_{1,3}$ as an induced subgraph, respectively is a line graph.

\section{References}

[1] N. Alon, D. Lokshtanov, and S. Saurabh. Fast FAST. In Proceedings of the 36th Colloquium of Automata, Languages and Programming (ICALP 2009), volume 5555 of Lecture Notes in Computer Science, pages 49-58. Springer, 2009.

[2] I. Bliznets, F. V. Fomin, M. Pilipczuk, and M. Pilipczuk. A subexponential parameterized algorithm for Interval Completion. CoRR, abs/1402.3473, 2014.

[3] I. Bliznets, F. V. Fomin, M. Pilipczuk, and M. Pilipczuk. A subexponential parameterized algorithm for Proper Interval Completion. In Proceedings of the 22nd Annual European Symposium on Algorithms (ESA 2014), volume 8737 of Lecture Notes in Computer Science, pages 173-184. Springer, 2014. 
[4] P. Burzyn, F. Bonomo, and G. Durán. NP-completeness results for edge modification problems. Discrete Applied Mathematics, 154(13):1824-1844, 2006.

[5] L. Cai. Fixed-parameter tractability of graph modification problems for hereditary properties. Information Processing Letters, 58(4):171-176, 1996.

[6] L. Cai and Y. Cai. Incompressibility of $H$-free edge modification. In Proceedings of the 8th International Symposium on Parameterized and Exact Computation (IPEC 2013), volume 8246 of Lecture Notes in Computer Science, pages 84-96. Springer, 2013.

[7] E. D. Demaine, F. V. Fomin, M. Hajiaghayi, and D. M. Thilikos. Subexponential parameterized algorithms on graphs of bounded genus and $H$-minor-free graphs. J. ACM, 52(6):866-893, 2005.

[8] R. G. Downey and M. R. Fellows. Parameterized complexity. Springer-Verlag, New York, 1999.

[9] P. G. Drange, F. V. Fomin, M. Pilipczuk, and Y. Villanger. Exploring subexponential parameterized complexity of completion problems. In Proceedings of the 31st International Symposium on Theoretical Aspects of Computer Science (STACS 2014), volume 25 of LIPIcs, pages 288-299. Schloss Dagstuhl Leibniz-Zentrum für Informatik, 2014.

[10] J. Edmonds. Paths, trees, and flowers. Canadian Journal of Mathematics, 17(3):449-467, 1965.

[11] J. Flum and M. Grohe. Parameterized Complexity Theory. Texts in Theoretical Computer Science. An EATCS Series. Springer-Verlag, Berlin, 2006.

[12] F. V. Fomin, S. Kratsch, M. Pilipczuk, M. Pilipczuk, and Y. Villanger. Tight bounds for parameterized complexity of Cluster Editing with a small number of clusters. Journal of Computer and System Sciences, 80(7):1430-1447, 2014.

[13] F. V. Fomin, D. Lokshtanov, N. Misra, and S. Saurabh. Planar F-deletion: Approximation, kernelization and optimal FPT algorithms. In Proceedings of the 53rd IEEE Annual Symposium on Foundations of Computer Science (FOCS 2012), pages 470-479. IEEE, 2012.

[14] F. V. Fomin, S. Saurabh, and Y. Villanger. A polynomial kernel for Proper Interval Vertex Deletion. SIAM Journal on Discrete Mathematics, 27(4):1964-1976, 2013.

[15] F. V. Fomin and Y. Villanger. Subexponential parameterized algorithm for minimum fill-in. SIAM Journal on Computing, 42(6):2197-2216, 2013.

[16] T. Gallai. Transitiv orientierbare graphen. Acta Mathematica Academiae Scientiarum Hungarica, 18(1-2):25-66, 1967.

[17] E. Ghosh, S. Kolay, M. Kumar, P. Misra, F. Panolan, A. Rai, and M. Ramanujan. Faster parameterized algorithms for deletion to split graphs. Algorithmica, 2013. Online first.

[18] S. Guillemot, F. Havet, C. Paul, and A. Perez. On the (non-)existence of polynomial kernels for $P_{l}$-free edge modification problems. Algorithmica, 65(4):900-926, 2013.

[19] J. Guo. Problem kernels for NP-complete edge deletion problems: Split and related graphs. In Proceedings of the 18th International Symposium on Algorithms and Computation (ISAAC 2007), volume 4835 of Lecture Notes in Computer Science, pages 915-926. Springer, 2007.

[20] R. Impagliazzo, R. Paturi, and F. Zane. Which problems have strongly exponential complexity? Journal of Computer and System Sciences, 63(4):512-530, 2001.

[21] Y. Jing-Ho, C. Jer-Jeong, and G. Chang. Quasi-threshold graphs. Discrete Applied Mathematics, 69(3):247-255, 1996.

[22] C. Komusiewicz and J. Uhlmann. Cluster editing with locally bounded modifications. Discrete Applied Mathematics, 160(15):2259-2270, 2012. 
[23] S. Kratsch and M. Wahlström. Two edge modification problems without polynomial kernels. In Proceedings of the 4th International Workshop on Parameterized and Exact Computation (IWPEC 2009), volume 5917 of Lecture Notes in Computer Science, pages 264-275. Springer, 2009.

[24] Y. Liu, J. Wang, and J. Guo. An overview of kernelization algorithms for graph modification problems. Tsinghua Science and Technology, 19(4):346-357, 2014.

[25] F. Mancini. Graph modification problems related to graph classes. PhD thesis, University of Bergen, Norway, 2008.

[26] R. M. McConnell and J. Spinrad. Modular decomposition and transitive orientation. Discrete Mathematics, 201(1-3):189-241, 1999.

[27] J. Nastos and Y. Gao. Familial groups in social networks. Social Networks, 35(3):439-450, 2013.

[28] M. Yannakakis. Edge-deletion problems. SIAM Journal on Computing, 10(2):297-309, 1981. 